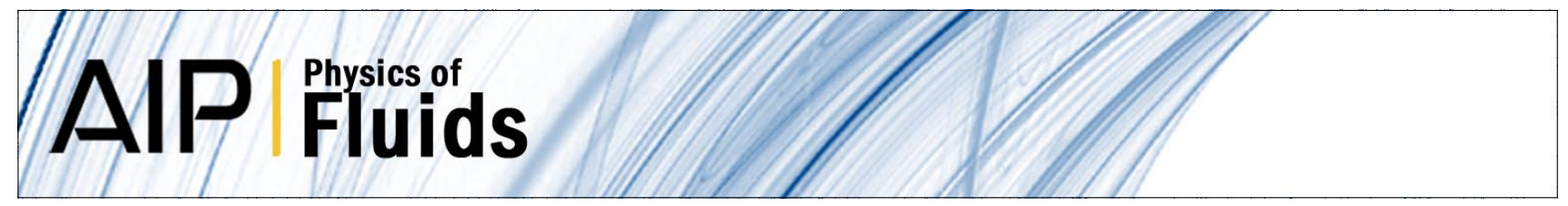

\title{
Numerical simulation of a gas-liquid flow in a fixed bed
}

Sangkyun Koo and Ashok S. Sangani

Citation: Phys. Fluids 13, 141 (2001); doi: 10.1063/1.1331314

View online: http://dx.doi.org/10.1063/1.1331314

View Table of Contents: http://pof.aip.org/resource/1/PHFLE6/v13/i1

Published by the American Institute of Physics.

\section{Related Articles}

Clouds of particles in a periodic shear flow

Phys. Fluids 24, 021703 (2012)

The dynamics of a vesicle in a wall-bound shear flow

Phys. Fluids 23, 121901 (2011)

A study of thermal counterflow using particle tracking velocimetry

Phys. Fluids 23, 107102 (2011)

Particle accumulation on periodic orbits by repeated free surface collisions

Phys. Fluids 23, 072106 (2011)

Drag force of a particle moving axisymmetrically in open or closed cavities

J. Chem. Phys. 135, 014904 (2011)

\section{Additional information on Phys. Fluids}

Journal Homepage: http://pof.aip.org/

Journal Information: http://pof.aip.org/about/about_the_journal

Top downloads: http://pof.aip.org/features/most_downloaded

Information for Authors: http://pof.aip.org/authors

\section{ADVERTISEMENT}

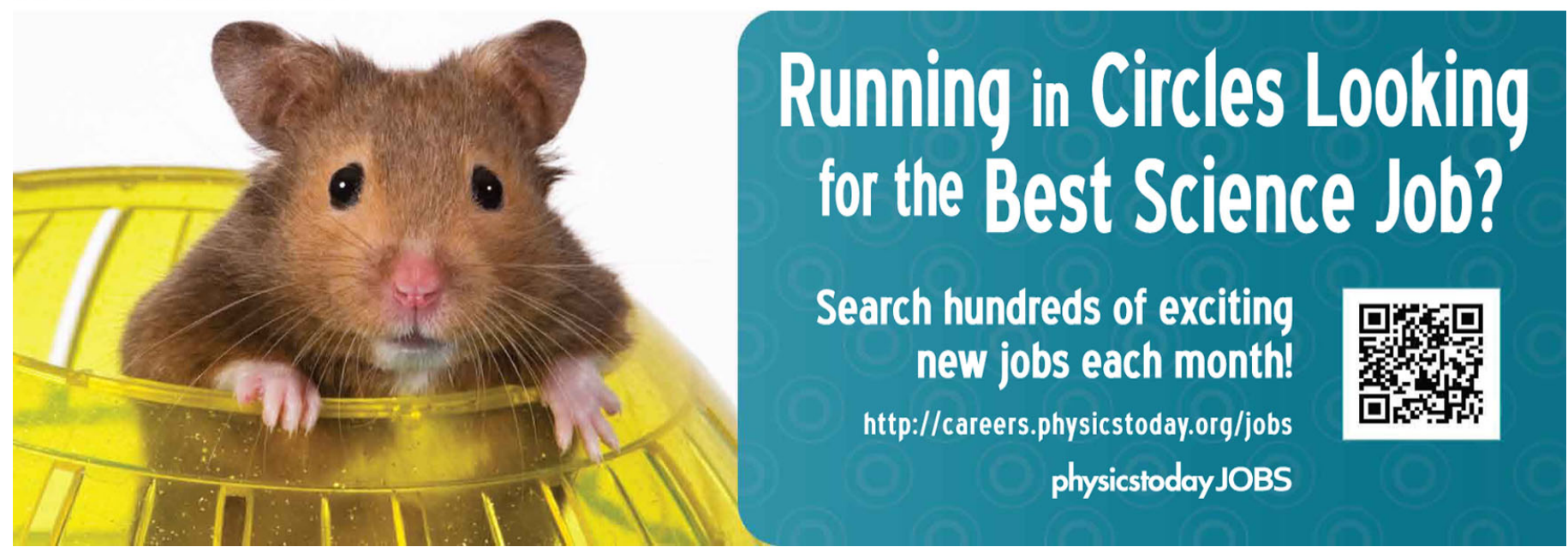




\title{
Numerical simulation of a gas-liquid flow in a fixed bed
}

\author{
Sangkyun Koo and Ashok S. Sangani ${ }^{\mathrm{a})}$ \\ Department of Chemical Engineering and Materials Science, Syracuse University, Syracuse, New York 13244
}

(Received 5 February 1999; accepted 10 October 2000)

\begin{abstract}
A countercurrent gas-liquid flow through a fixed bed of spherical particles is examined numerically by solving the particle-scale equations governing the gas and liquid flows. The liquid is assumed to flow along the surface of the particles forming a thin film. The case of small gas flow rates is examined in detail first. In this limit the presence of the liquid film increases the gas pressure drop over its value for a dry bed by three mechanisms: The liquid film makes the apparent size of the particles larger, decreases the pore space for the gas flow, and, with its velocity pointing opposite to the mean gas flow, increases the apparent velocity of the gas compared with the particle surface. The excess pressure drop is determined for both periodic and random arrangements of particles. Next, the case of high gas flow rates where the traction exerted by the gas at the gas-liquid interface is comparable to the weight of the liquid film is examined. In this regime the liquid holdup increases with the gas flow rate and the pressure drop-gas velocity relation is nonlinear. The results of numerical simulations are compared with approximate models and it is shown that a simple capillary model yields reasonably accurate predictions for the liquid holdup and gas pressure drop. (C) 2001 American Institute of Physics. [DOI: 10.1063/1.1331314]
\end{abstract}

\section{INTRODUCTION}

Fixed beds of particles are widely employed in chemical industry for absorption, stripping, distillation, and other separation processes, and as reactors to provide efficient contact between liquid and gas (or vapor) phases. Typically, the gas flows upward and the liquid flows downward under the action of gravity through the bed. An important problem in these processes is to predict the gas pressure drop across the bed and the liquid phase volume fraction (holdup) as functions of the gas and liquid flow rates and the particle volume fraction. Another problem of interest is the prediction of critical gas flow rate above which the liquid starts accumulating at the top of the bed, a condition known as the flooding.

The case of single-phase flow through a fixed bed of particles has been examined extensively in the literature, both theoretically as well as experimentally. Probably the first systematic approach was due to Carman ${ }^{1}$ who modeled the void space in the fixed bed by straight capillaries whose diameter is taken to be a function of the volume fraction of the particles and the size of particles. The pressure drop in the fluid as it moves through the bed as calculated with this model with one adjustable parameter is shown to compare very well with the experimentally measured pressure drop in packed beds of spherical particles when the Reynolds number based on the particle diameter and average velocity of the fluid is less than about 10. The pressure drop at larger Reynolds number can be evaluated with an empirical extension of the above analysis using the so-called Ergun equation. ${ }^{2}$

In recent years analytical efforts have been directed at

\footnotetext{
a) Author to whom correspondence should be addressed. Telephone: 315-443-4502; Fax: 315-443-2559; electronic mail: asangani@mailbox.syr.edu
}

computing the pressure drop-velocity relationship for welldefined geometry of fixed beds. For example, Sorensen and Stewart, ${ }^{3}$ Zick and Homsy, ${ }^{4}$ and Sangani and Acrivos ${ }^{5}$ considered the case of equal-sized spheres arranged in a periodic array while Ladd $^{6}$ and Mo and Sangani ${ }^{7}$ considered the case of random arrays. These studies were limited to small Reynolds numbers for which the fluid inertia is negligible. The effect of inertia at moderately large Reynolds numbers (up to about 100) have been examined for the two-dimensional case of periodic as well as random arrays of infinitely long fixed cylinders by Ghaddar ${ }^{8}$ and Koch and Ladd. ${ }^{9}$

In contrast to the above, rigorous analytical studies solving the equations governing the gas and liquid motion at the particle-scale are lacking. Instead the focus has been on using volume-averaged macroscale equations to understand various flow regime transitions in fixed beds including the onset of flooding in the countercurrent gas-liquid flow ${ }^{10}$ and the steady, uniform flow to pulsing in concurrent gas-liquid flows. ${ }^{11,12}$ Although the constitutive relations and the dependence of forces acting on the liquid and gas phases on the volume fractions of the individual phases are based on empirical correlations, these studies have been generally successful in explaining, at least qualitatively, many of the macroscopic features observed in these systems. $\mathrm{Ng}^{13}$ on the other hand explained the origin of various flow regime transitions with the help of a semirigorous microscale model of fixed bed. Observations on various flow regimes for gasliquid flows through fixed bed of particles may be found in the review articles by de Santos, Melli, and Scriven. ${ }^{14}$

The present study is, to our knowledge, the first attempt at directly solving the gas-liquid flow through an assemblage of fixed particles. Because of the complexity of these flows in general the problem we shall examine is considerably idealized. Nevertheless, it is hoped that such a 
microscale-based approach may help develop, for example, better expressions for the forces on the individual phases and may provide better insight into instability phenomena that may originate at particle scale for which the applicability of the averaged-equations is questionable.

In general, the phenomenon of gas-liquid flows through a fixed bed of particles is influenced by a number of parameters even when the bed consists of equal-sized spheres and the Reynolds number based on the average gas velocity is small. At very low gas flow rates the liquid trickles down from one particle to the next down the bed with the liquid flow governed by the wetting characteristics of the particles, gravity, the geometry of the bed, and the nature of liquid distributor. We shall consider here the case of wetting liquids with low enough flow rates such that the liquid film around each particle can be regarded as small compared with the size of the particles. In principle, the liquid flow distribution at low gas flow rates can be computed given the position of the particles and the liquid distributor geometry but, to keep the number of parameters to a minimum, we shall limit the flow distribution to two special cases. In the first case, the liquid arrives at the top (the north pole) of each particle, flows down under the influence of gravity along the particle surface, and leaves the particle from its lowest point (the south pole). The liquid film in this case is nonuniform with the maximum thickness occurring at the north and south poles of the particle. The second case corresponds to a uniform film thickness. While one expects the liquid flow to be unaffected by the presence of the gas when the flow rate of the latter is small, the gas flow rate will be influenced by the presence of the liquid film around each particle and we account for this in our analysis using a domain perturbation technique. The presence of the liquid increases the pressure drop in the gas by three mechanisms: first, the liquid film appears to make particle bigger in size and this causes an increase in the drag exerted by the gas on the particle; second, at finite volume fractions of the fixed particles, the effect of film is to effectively decrease the pore space for the gas flow which in turn leads to a greater drag force; and third, the downward moving liquid film at the particle surface makes the gas appear to have a negative slip velocity at the particle surface causing thereby an effective increase in the speed of the gas relative to the particles. We use a method of multipole expansion to determine these effects separately for both random and periodic arrays of spheres.

The preceding discussion applies to the low gas flow rates where the liquid flow and film thickness are governed by the gravity force acting on it and the viscous stresses at the solid-liquid interface. The resulting gas pressure drop, although different from that for the dry bed, varies linearly with the superficial gas velocity owing to the small Reynolds number. At high gas flow rates the shear stress caused by the gas at the gas-liquid interface will also affect the liquid film thickness. The average film thickness increases with the increasing gas flow rate and the resulting pressure dropvelocity relation becomes nonlinear in this gas flow regime referred to in the chemical engineering literature as the loading regime. Fixed beds are usually operated in this regime since it yields higher residence time for the liquid in the bed.
We use a finite difference method to determine the liquid film thickness and a boundary perturbation technique together with the method of multipole expansion to determine the gas velocity distribution. The steady state solutions of the microscale equations are determined and compared with the predictions based approximate models and the averagedequations used in previous investigations. The agreement with the approximate models is seen to be quite good.

The rest of the paper is organized as follows: Sec. II gives the equations governing the liquid and gas velocities. Section III considers in detail the low gas flow regime while Sec. IV examines the loading regime. Finally, Sec. V summarizes some of the important findings of the work.

\section{FORMULATION OF THE PROBLEM}

As mentioned in the Introduction, we shall assume that the liquid wets the particles completely and thereby forms a film around each particle in a fixed bed consisting of $N$ particles placed within a unit cell of a periodic array. The liquid may also form drops that may travel from one particle to the next in the bed. The effect of these drops on the gas flow will be neglected in the present analysis. This approximation is justified when the drop size is small compared to the size of the particles. We shall also neglect the effect of inertia in describing the gas flow. This may not be a reasonable approximation for commercial packed beds in which the particle size is often of order of $1 \mathrm{~cm}$ but the case of small Reynolds numbers is the easiest to treat analytically and may be expected to apply up to Reynolds number of about 10 . The results obtained here may be adjusted, perhaps in an $a d$ hoc manner by adding an Ergun correction typical of single phase flows, before they may be applied for predicting pressure drop or liquid holdup.

For small Reynolds number flows the gas velocity satisfies the well-known Stokes equations of motion. The boundary conditions for the gas and liquid flows are the usual kinematic and dynamic boundary conditions at the gasliquid interface, the no-slip condition at the particle surface, and the periodicity conditions for the gas flow. These equations are supplemented with additional conditions specifying the total gas and liquid flow rates through the bed.

\section{LOW GAS FLOW RATES}

Let us first consider the case of gas flow rates for which the traction exerted by gas at the gas-liquid interface is negligible. For the gas to affect negligibly the liquid flow due to gravitational acceleration $\mathbf{g}$ acting on a film of thickness $\delta$, we must have $\rho_{l} g \delta \gg f_{s}$ where $\rho_{l}$ is the density of the liquid and $f_{s}$ is the magnitude of the shear stress produced by the gas at the gas-liquid interface. For Stokes flow conditions $f_{s}$ is $O\left(\mu_{g} U_{g} / a\right), a$ being the radius of the particle, $\mu_{g}$ the gas viscosity, and $U_{g}$ the superficial gas velocity through the bed. Thus, the case of low gas flow rates corresponds to $U_{g} \ll U_{g l}$ with

$$
U_{g l} \equiv \frac{\rho_{l} g a \delta_{0}}{\mu_{g}},
$$


where $\delta_{0}$ is the characteristic liquid film thickness [defined more precisely later, cf. (4)]. We shall refer to $U_{g l}$ as the loading velocity as it represents roughly the gas velocity above which the liquid flow and liquid phase holdup will be affected by the gas flow and the gas pressure drop-velocity relation will be nonlinear even in the Stokes flow regime. This regime will be considered in more detail in the next section.

For $U_{g} \ll U_{g l}$ we can first determine the liquid velocity distribution and use it subsequently to provide the boundary conditions for the gas flow. As mentioned in the Introduction, the liquid flow distribution depends in general on a number of factors including wetting characteristics, the nature of the liquid distributor, and the spatial configuration of particles. We shall consider here the simplest case in which the liquid flow on each particle is the same and governed by gravity. When the liquid film thickness $\delta$ is small compared with $a$, the liquid flow caused by the action of gravity gives rise to a quadratic profile

$$
\mathbf{u}_{l}=\frac{\rho_{l} g_{\theta}}{2 \mu_{l}}\left(2 y \delta-y^{2}\right) \mathbf{e}_{\theta},
$$

where $y$ is the distance from the surface of the particle, $\mathbf{e}_{\theta}$ is the unit vector along the polar angle $\theta$ measured from the $x_{1}$-axis, the direction opposite to the mean liquid flow, and $g_{\theta}=g \sin \theta$. The film thickness $\delta$ depends on the total liquid flow rate. If the liquid enters at $\theta=0$ and leaves from $\theta$ $=\pi$ at a steady volumetric flow rate $Q_{l}$, then we have, by integrating the velocity over the azimuthal angle $\varphi$ and $y$,

$$
Q_{l}=2 \pi a \sin \theta \int_{0}^{\delta}\left|\mathbf{u}_{l}\right| d y=\frac{\rho_{l} g}{3 \mu_{l}} 2 \pi a \delta^{3} \sin ^{2} \theta .
$$

The film thickness is then given by

$$
\delta=\delta_{0}(\sin \theta)^{-2 / 3} \quad \text { with } \quad \delta_{0} \equiv\left(\frac{3 \mu_{l} Q_{l}}{2 \pi a \rho_{l} g}\right)^{1 / 3} .
$$

We note that the liquid film thickness diverges as $\theta \rightarrow 0$ and $\theta \rightarrow \pi$. This is a consequence of the assumption that all the liquid arrives at $\theta=0$ and leaves the surface from $\theta=\pi$ where the cross-sectional areas are essentially zero and the gravity force for flow along the surface is zero. If we assume that not all of the liquid arrives at $\theta=0$ but over a small portion of the sphere with $\theta<\theta_{0}$ and leaves the sphere from $\pi-\theta_{0}<\theta<\pi$, then the film thickness will be finite everywhere on the sphere. Accordingly, we require that the volumetric flow rate for $\theta<\theta_{0}$ be given by

$$
Q_{l}\left(\sin \theta / \sin \theta_{0}\right)^{2} \quad \text { for } \theta<\theta_{0}
$$

with a similar expression applicable to $\pi-\theta_{0}<\theta<\pi$. With this flow distribution the film thickness and the velocity of the liquid at the gas-liquid interface $y=\delta$ are given by

$$
\delta=\delta_{0} H_{0}(\theta), \quad u_{\theta}=A \sin \theta H_{0}^{2},
$$

where

$$
\begin{aligned}
& H_{0}=(\sin \theta)^{-2 / 3} \text { for } \theta_{0}<\theta<\pi-\theta_{0}, \\
& H_{0}=\left(\sin \theta_{0}\right)^{-2 / 3} \text { for } \theta<\theta_{0} \text { and } \pi-\theta_{0}<\theta<\pi .
\end{aligned}
$$

The characteristic liquid velocity $A$ is given by

$$
A=\frac{\rho_{l} g \delta_{0}^{2}}{2 \mu_{l}} .
$$

In the above calculations we have neglected the effect of surface tension and the gas density. While the latter could be accounted for by simply replacing $\rho_{l}$ with the density difference $\rho_{l}-\rho_{g}$ in the above expressions, the former, i.e., the neglect of surface tension, calls for some discussion. When the surface tension effects are important the pressure inside the liquid will vary as the curvature of the film changes along the surface of the particle. With the pressure in the gas phase set to zero, the liquid flow is now driven by the tangential component of $\rho_{l} \mathbf{g}-\nabla p_{l}$. Thus, $\rho_{l} g_{\theta}$ in (2) must be replaced by

$$
\rho_{l} g_{\theta}-\frac{1}{a} \frac{\partial p_{l}}{\partial \theta},
$$

where $p_{l}$ is the pressure difference across the gas-liquid interface which for thin films may be evaluated using

$$
p_{l}=\sigma \nabla \cdot \mathbf{n}=\frac{2 \sigma}{a}\left[1-\frac{\delta}{a}+\frac{1}{2 a} \nabla_{s}^{2} \delta+O\left(\delta^{2} / a^{2}\right)\right],
$$

where $\sigma$ is the surface tension, $\mathbf{n}$ is the unit normal vector at the gas-liquid interface pointing into the gas phase, and $\nabla_{s}^{2}$ is the surface Laplacian on a unit sphere, i.e.,

$$
\nabla_{s}^{2}=\frac{1}{\sin \theta} \frac{\partial}{\partial \theta}\left(\sin \theta \frac{\partial}{\partial \theta}\right)+\frac{1}{\sin ^{2} \theta}\left(\frac{\partial^{2}}{\partial \varphi^{2}}\right) .
$$
(3),

Now the liquid volumetric flow is given by, in lieu of

$$
Q_{l}=\frac{\rho_{l} g \delta^{3}}{3 \mu_{l}} 2 \pi a\left[\sin ^{2} \theta+\frac{\sigma \delta_{0}}{a^{3} \rho_{l} g} \sin \theta \frac{1}{\delta_{0}} \frac{\partial}{\partial \theta}\left\{-2 \delta+\nabla_{s}^{2} \delta\right\}\right] .
$$

Thus, when the surface tension effect is important it is necessary to integrate the nonlinear third-order differential equation (12) together with suitable boundary conditions for determining the liquid film thickness distribution instead of the simple, algebraic equation (3). Fortunately the nondimensional surface tension, $\sigma \delta_{0} / a^{3} \rho_{l} g$, multiplying the derivative terms is very small unless the particle is smaller than 1 $\mathrm{mm}$ in radius. For example, for an air-water system with $\delta_{0} / a=0.05, \sigma=70 \mathrm{~g} \mathrm{~cm} / \mathrm{s}$, and $a=3 \mathrm{~mm}$, the above nondimensional number is less than 0.04 . Neglecting the surface tension term altogether from (12) will not be uniformly valid approximation since the third-order differential equation will then be simply reduced to an algebraic equation but this approximation will break down only near the poles $\theta=0$ and $\pi$ where, how the liquid arrives or leaves the surface would need to be specified in more detail to determine $\delta$. We expect the simple expression (4) to hold for most $\theta$ values except near the poles.

With the liquid velocity and film thickness determined we now turn to the problem of determining the gas velocity field. The boundary condition for the gas motion is the continuity of velocity at the gas-liquid interface. We shall treat 
$\delta_{0} / a$ as a small parameter and determine the gas velocity field using a domain perturbation technique correct to $O\left(\delta_{0} / a\right)$. The liquid velocity and film thickness determined above are valid only to $O(1)$. The analysis of the liquid flow is continued to $O\left(\delta_{0} / a\right)$ in Appendix A. Let us expand the gas velocity in powers of $\delta_{0} / a$ as

$$
\mathbf{u}_{g}=\mathbf{u}^{(0)}+\left(\delta_{0} / a\right) \mathbf{u}^{(1)}+\cdots .
$$

Now, as shown in Appendix A, the continuity of the velocity at the gas-liquid interface yields the following boundary conditions for $\mathbf{u}^{(0)}$ and $\mathbf{u}^{(1)}$ :

$$
\begin{aligned}
\mathbf{u}^{(0)}= & A \sin \theta H_{0}^{2} \mathbf{e}_{\theta} \quad \text { at } r=a, \\
\mathbf{u}^{(1)}= & -a H_{0} \frac{\partial \mathbf{u}^{(0)}}{\partial r}+A\left[(3 / 4) \sin \theta H_{0}^{3}\right. \\
& \left.-(1 / 3) \cos \theta H_{0}^{2} H_{0}^{\prime}\right] \mathbf{e}_{\theta} \quad \text { at } r=a,
\end{aligned}
$$

where $H_{0}$ is the scaled liquid film thickness given by (7).

To model an infinitely extended fixed bed of particles we follow the standard practice and assume the bed to consist of a periodic array with each unit cell of the array containing $N$ spherical particles whose positions are generated using a specified spatial distribution law. The above boundary conditions (14)-(15) must be satisfied on the surface of each particle. In addition, the velocity must be spatially periodic. Finally, an additional constraint to be satisfied is

$$
\frac{1}{\tau} \int_{\mathcal{V}_{g}} \mathbf{u}_{g} d V=\mathbf{U}_{g},
$$

where $\mathbf{U}_{g}$ is the superficial gas velocity through the bed, $\tau$ is the volume of the unit cell, and $\mathcal{V}_{g}$ is the volume occupied by the gas within the basic unit cell.

We shall use the method outlined in Mo and Sangani ${ }^{7}$ for determining $\mathbf{u}^{(0)}$ and $\mathbf{u}^{(1)}$. Briefly, the method consists of writing a formal solution of Stokes equations of motion in terms of derivatives of a periodic fundamental singular solution of Stokes equations. This formal solution containing a number of undetermined coefficients satisfies the periodicity and the governing Stokes equations of motion. The coefficients are subsequently determined by expanding the formal solution around the surface of each particle and satisfying the boundary conditions on the particle surface. The expansion near a representative particle $\alpha$ is expressed in terms of spherical harmonics according to the well-known Lamb's solution, ${ }^{15}$

$$
\mathbf{u}(\mathbf{x})=\sum_{n=-\infty}^{\infty}\left[\left(c_{n} r^{2} \nabla p_{n}^{\alpha}+b_{n} \mathbf{r} p_{n}^{\alpha}\right)+\nabla \times\left(\mathbf{r} \chi_{n}\right)+\nabla \phi_{n}^{\alpha}\right],
$$

with $\mathbf{r}=\mathbf{x}-\mathbf{x}^{\alpha}$ and

$$
c_{n}=\frac{n+3}{2(n+1)(2 n+3)}, \quad b_{n}=\frac{-n}{(n+1)(2 n+3)} .
$$

Here, $p_{n}, \phi_{n}$, and $\chi_{n}$ in (17) are the spherical harmonics of order $n$. The harmonics of negative order are singular at $\mathbf{r}$ $=\mathbf{0}$, and we express them as

$$
p_{-n-1}^{\alpha}=\sum_{m=0}^{n}\left(P_{n m}^{s, \alpha} Y_{n m}+\widetilde{P}_{n m}^{s, \alpha} \widetilde{Y}_{n m}\right) r^{-n-1} \quad(n>0) .
$$

(Note that $p_{-1}=\chi_{-1}=\phi_{-1}=0$.) In (19) $P_{n m}^{s, \alpha}$ and $\widetilde{P}_{n m}^{s, \alpha}$ are the coefficients of the singular harmonics and

$$
Y_{n m}=P_{n}^{m}(\cos \theta) \cos m \varphi, \quad \tilde{Y}_{n m}=P_{n}^{m}(\cos \theta) \sin m \varphi
$$

are the surface harmonics with $P_{n}^{m}$ being the associated Legendre polynomial and $\theta$ and $\varphi$ the polar and azimuthal angles defined by $x_{1}-x_{1}^{\alpha}=r \cos \theta, x_{2}-x_{2}^{\alpha}=r \sin \theta \cos \varphi$, and $x_{3}-x_{3}^{\alpha}=r \sin \theta \sin \varphi$. The singular harmonics $\chi_{-n-1}$ and $\phi_{-n-1}$ are likewise expressed in terms of coefficients $T_{n m}^{s, \alpha}, \widetilde{T}_{n m}^{s, \alpha}, \Phi_{n m}^{s, \alpha}$, and $\widetilde{\Phi}_{n m}^{s, \alpha}$

The harmonics with non-negative $n$ are expressed as

$$
p_{n}^{\alpha}=\sum_{m=0}^{n}\left(P_{n m}^{r, \alpha} Y_{n m}+\widetilde{P}_{n m}^{r, \alpha} \widetilde{Y}_{n m}\right) r^{n} \quad n \geqslant 0
$$

with similar expressions for $\chi_{n}^{\alpha}$ and $\phi_{n}^{\alpha}$.

To satisfy the boundary conditions for the velocity at $r$ $=a$ it is convenient to use

$$
\begin{aligned}
& u_{r}=\sum_{n=-\infty}^{\infty}\left(n c_{n}+b_{n}\right) r p_{n}+(n / r) \phi_{n}, \\
& \nabla_{s} \cdot \mathbf{u}_{s}=-\sum_{n=-\infty}^{\infty} n(n+1)\left[c_{n} r p_{n}+\phi_{n} / r\right], \\
& \mathbf{e}_{r} \cdot\left(\nabla \times \mathbf{u}_{s}\right)=\sum_{n=-\infty}^{\infty} n(n+1) \chi_{n} / r,
\end{aligned}
$$

where $u_{r}$ is the radial component of the velocity, $\mathbf{u}_{s}=u_{\theta} \mathbf{e}_{\theta}$ $+u_{\phi} \mathbf{e}_{\phi}$ is the tangential velocity at the surface of the sphere, and

$$
\nabla=\mathbf{e}_{r} \frac{\partial}{\partial r}+\frac{1}{r} \nabla_{s}
$$

The expressions given above apply equally well to $\mathbf{u}^{(0)}$ and $\mathbf{u}^{(1)}$. The solutions for these two quantities differ mainly through the boundary conditions [cf. (14) and (15)]. Let us denote by $\mathbf{v}$ the velocity distribution at $r=a$. Then $\mathbf{v}$ for the $\mathbf{u}^{(0)}$ and $\mathbf{u}^{(1)}$ problems are given by the right-hand sides of (14) and (15), respectively.

Let us expand $v_{r}, \nabla_{s} \cdot \mathbf{v}_{s}$ and $\mathbf{e}_{r} \cdot\left(\nabla \times \mathbf{v}_{s}\right)$ also in spherical surface harmonics. Thus, we write

$$
v_{r}=\sum_{n=0}^{\infty} \sum_{m=0}^{n}\left[\left(v_{r}\right)_{n m} Y_{n m}+\left(\tilde{v}_{r}\right)_{n m} \tilde{Y}_{n m}\right]
$$

Similar expressions are written for $\nabla_{s} \cdot \mathbf{v}_{s}$ and $\mathbf{e}_{r} \cdot\left(\nabla \times \mathbf{v}_{s}\right)$ in terms of coefficients denoted by $\left[\nabla_{s} \cdot \mathbf{v}_{s}\right]_{n m}, \quad\left[\mathbf{e}_{r} \cdot(\nabla\right.$ $\left.\times \mathbf{v}_{s}\right]_{n m}$, and the corresponding quantities with tilde. The coefficients $\left(v_{r}\right)_{n m},\left(\nabla_{s} \cdot \mathbf{v}_{s}\right)$, etc. appearing in these expansions can be determined by integrating the functions multiplied by surface harmonics over a surface of a unit sphere. Thus, for example, since $\nabla_{s} \cdot \mathbf{v}_{s}^{(0)}=(2 A / 3) \cos \theta H_{0}^{2}$, we have 


$$
\left(\nabla_{s} \cdot \mathbf{v}_{s}\right)_{n m}^{(0)}=\frac{2 A}{3} \frac{\int_{\theta=0}^{\pi} \int_{0}^{2 \pi} \sin \theta \cos \theta H_{0}^{2} Y_{n m}(\theta, \varphi) d \theta d \varphi}{\int_{0}^{\pi} \int_{0}^{2 \pi} Y_{n m}^{2} \sin \theta d \theta d \varphi} .
$$

The surface of the sphere was discretized into a number of triangular elements to evaluate the integrals appearing in the expressions such as above numerically.

Now using the orthogonality of surface harmonics and the expressions (22)-(24) the boundary conditions at $r=a$ yield

$$
\begin{aligned}
& \quad\left[-(n+1) c_{-n-1}+b_{-n-1}\right] P_{n m}^{s, \alpha} a^{-n}+\left[n c_{n}+b_{n}\right] P_{n m}^{r, \alpha} a^{n+1} \\
& \quad-(n+1) \Phi_{n m}^{s, \alpha} a^{-n-2}+n \Phi_{n m}^{r} a^{n-1}=\left(v_{r}\right)_{n m}, \\
& -n(n+1)\left[P_{n m}^{s, \alpha} a^{-n}+P_{n m}^{r, \alpha} a^{n+1}+\Phi_{n m}^{s, \alpha} a^{-n-2}+\Phi_{n m}^{r, \alpha} a^{n-1}\right] \\
& \quad=\left(\nabla_{s} \cdot \mathbf{v}_{s}\right)_{n m}, \\
& -n(n+1)\left[T_{n m}^{s, \alpha} a^{-n-2}+T_{n m}^{r, \alpha} a^{n-1}\right]=\left[\mathbf{e}_{r} \cdot\left(\nabla \times \mathbf{v}_{s}\right)\right]_{n m},
\end{aligned}
$$

plus similar equations involving the coefficients of $\widetilde{Y}_{n m}$.

The singular coefficients in the above equations represent the effect of particle $\alpha$, whereas the regular coefficients represent the effect of other particles and the imposed flow. As mentioned earlier, Mo and Sangani ${ }^{7}$ wrote the expression for the velocity in terms of fundamental periodic singular solution of Stokes equations $v_{i j}$ as

$$
u_{i}(\mathbf{x})=U_{i}^{*}+\sum_{\alpha=1}^{N} \mathcal{G}_{j}^{\alpha} v_{i j}\left(\mathbf{x}-\mathbf{x}^{\alpha}\right),
$$

where $\mathcal{G}_{j}^{\alpha}$ is a differential operator defined in terms of the singular coefficients $P_{n m}^{s, \alpha}, \Phi_{n m}^{s, \alpha}$, etc., in such a way that $\mathcal{G}_{j}^{\alpha} v_{i j}\left(\mathbf{x}-\mathbf{x}^{\alpha}\right)$ corresponds exactly to the singular terms in (17) as $\mathbf{x} \rightarrow \mathbf{x}^{\alpha}$. The coefficients of the regular terms in the Lamb's solution, i.e., $P_{n m}^{r, \alpha}, T_{n m}^{r, \alpha}$, etc., are related to various derivatives of the regular part of $\mathbf{u}$ at $\mathbf{x}=\mathbf{x}^{\alpha}$. The reader is referred to Mo and Sangani ${ }^{7}$ for more details.

Finally, $\mathbf{U}^{*}$ can be shown to be the same as the superficial gas velocity. Since the integrals of $v_{i j}$ and its derivatives over the unit cell vanish, integrating (31) over the volume occupied by the gas gives

$$
\mathbf{U}_{g}=\mathbf{U}^{*}-\frac{1}{\tau} \sum_{\alpha=1}^{N} \int_{\mathcal{V}^{\alpha}} \mathbf{u} d V=\mathbf{U}^{*}-\frac{1}{\tau} \sum_{\alpha=1}^{N} \int_{S_{g l}^{\alpha}} \mathbf{n} \cdot \mathbf{u r} d A,
$$

where $\mathcal{V}^{\alpha}$ is the volume occupied by the particle $\alpha$ and the surrounding liquid film, $\mathbf{r}=\mathbf{x}-\mathbf{x}^{\alpha}, S_{g l}^{\alpha}$ is the gas-liquid interface enclosing particle $\alpha$. Note that use has been made of the identity $\mathbf{u}=\nabla \cdot(\mathbf{u r})$ together with the divergence theorem to convert the volume integral into the surface integral. Now $\mathbf{u} \cdot \mathbf{n}=0$ at the gas-liquid interface proving thereby that $\mathbf{U}^{*}$ $=\mathbf{U}_{g}$.

The coefficients $P_{n m}^{s, \alpha}$, etc., were expanded in a series in

\begin{tabular}{|c|c|c|c|c|c|c|c|}
\hline \multirow[b]{2}{*}{$\phi$} & \multirow[b]{2}{*}{$N_{s}$} & \multirow[b]{2}{*}{$K$} & \multirow[b]{2}{*}{$f_{1}$} & \multicolumn{2}{|c|}{$f_{2}$} & \multicolumn{2}{|c|}{$f_{3}$} \\
\hline & & & & $\theta_{0}=\pi / 20$ & $\theta_{0}=\pi / 40$ & $\theta_{0}=\pi / 20$ & $\theta_{0}=\pi / 40$ \\
\hline 0.001 & 7 & 1.22 & 1.34 & 0.84 & 0.84 & -1.73 & -5.77 \\
\hline 0.005 & 7 & 1.43 & 1.57 & 0.84 & 0.84 & -1.73 & -5.75 \\
\hline 0.1 & 7 & 3.76 & 3.45 & 0.77 & 0.77 & -1.60 & -5.38 \\
\hline 0.2 & 7 & 7.05 & 5.27 & 0.70 & 0.70 & -1.46 & -4.93 \\
\hline 0.3 & 7 & 12.79 & 7.54 & 0.62 & 0.62 & -1.29 & -4.39 \\
\hline \multirow[t]{2}{*}{0.4} & 7 & 23.91 & 10.52 & 0.53 & 0.53 & -1.11 & -3.78 \\
\hline & 9 & 23.91 & 10.54 & 0.53 & 0.53 & -1.11 & -3.78 \\
\hline \multirow[t]{2}{*}{0.5} & 9 & 47.96 & 14.63 & 0.43 & 0.43 & -0.91 & -3.10 \\
\hline & 11 & 47.96 & 14.63 & 0.43 & 0.43 & -0.91 & -3.10 \\
\hline \multirow[t]{2}{*}{0.6} & 9 & 107.60 & 20.25 & 0.32 & 0.32 & -0.68 & -3.10 \\
\hline & 11 & 107.53 & 20.25 & 0.32 & 0.32 & -0.69 & -2.36 \\
\hline \multirow[t]{3}{*}{0.7} & 9 & 277.27 & 26.52 & 0.21 & 0.21 & -0.45 & -1.57 \\
\hline & 11 & 279.29 & 26.62 & 0.21 & 0.21 & -0.45 & -1.55 \\
\hline & 13 & 280.45 & 26.81 & 0.21 & 0.21 & -0.45 & -1.54 \\
\hline
\end{tabular}
$\delta / a$ as $P_{n m}=P_{n m}^{(0)}+\left(\delta_{0} / a\right) P_{n m}^{(1)}+\cdots$ and (28)-(30) were rearranged and truncated as in Mo and Sangani ${ }^{7}$ to solve for these coefficients. The force on particle $\alpha$ in the $x_{1}$-direction (antigravity direction) is related to $P_{10}^{s, \alpha}$ by

$$
F^{\alpha}=-4 \pi \mu_{g} P_{10}^{s, \alpha} .
$$

TABLE I. The coefficients $K$ and $f_{1}-f_{3}$ for the face-centered cubic array.

The above represents the force exerted by gas at the gasliquid interface. To calculate the total force on the particle one must add the weight of the liquid film surrounding the particle. The pressure drop in the gas will be related to the force exerted by the gas, i.e., that given by (33) [cf. (35)]. For the sake of brevity therefore we shall refer to the above force by gas on the gas-liquid interface as the force on the particle.

Results: The results for the average force on a particle are expressed in terms of coefficients $K$ and $f_{1}-f_{3}$ defined by

$F=6 \pi \mu_{g} a U_{g} K(\phi)\left[1+\left(\delta_{0} / a\right) f_{1}+\frac{A}{U_{g}}\left\{f_{2}+\left(\delta_{0} / a\right) f_{3}\right\}\right]$,

where $K$ represents the nondimensional drag on the particle in a dry bed, $f_{1}$ represents the effect of the finite thickness of the liquid film on the force exerted on the particle by the gas moving with finite mean velocity, and $f_{2}$ and $f_{3}$ represent the effect of downward motion of liquid. Recall that $A$ is the characteristic liquid velocity at the gas-liquid interface. Note also that the force on a particle is nonzero even when there is no net gas flow through the bed. The downward moving liquid drags along with it some gas and to compensate for this the gas away from the surface of the sphere must move upwards causing a net nonzero force. The pressure gradient in the gas is related to the force by

$$
-\frac{d P}{d x_{1}}=n F=\frac{3 \phi_{s}}{4 \pi a^{3}} F,
$$

where $n$ is the number of spheres per unit volume of the bed and $\phi_{s}$ is the volume fraction occupied by the spheres. The results for $K$ and $f_{1}-f_{3}$ for the case of face-centered cubic arrays, which permits the largest range of particle volume fraction, are given in Table I.

The results for the dry bed pressure drop, or equivalently $K$, have been obtained previously for periodic arrays by Zick and Homsy ${ }^{4}$ and Sangani and Acrivos ${ }^{5}$ and for random arrays by Ladd ${ }^{6}$ and Mo and Sangani. ${ }^{7}$ Our results for periodic as well as random arrays for $K$ shown in Fig. 1 were found to 


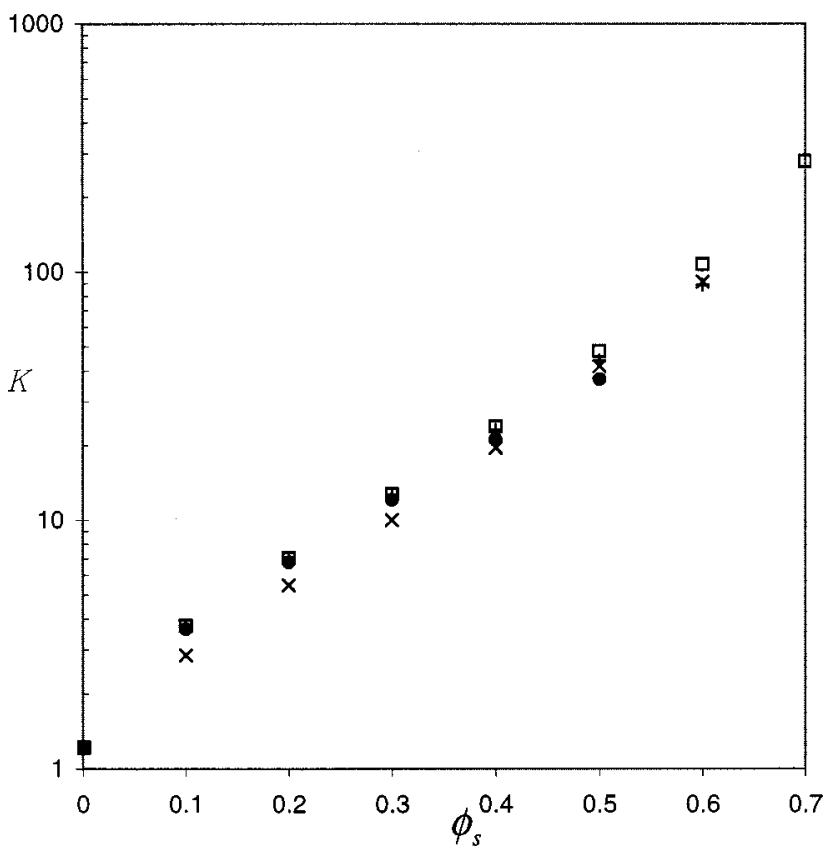

FIG. 1. The dry bed drag coefficient $K$ as a function of $\phi_{s}$. The filled circles are the results for the simple cubic arrays, pluses for the body-centered cubic arrays, squares for the face-centered cubic arrays, and crosses for the random arrays of spheres.

be in excellent agreement with the results obtained by these investigators. The results for random arrays were obtained by averaging over 20 configurations generated using a hardsphere molecular dynamics code which employed 16 particles per unit cell.

The results for $f_{1}$ will be compared with an approximate relation obtained by assuming that the main effect of the film is to increase the apparent size of the particle and the apparent particle volume fraction. Thus an approximate relation for the force on a particle is obtained by writing

$$
\begin{aligned}
F_{\text {approx }}= & 6 \pi \mu_{g} U_{g}\left(a+\delta_{0}\right) K\left(\phi_{s}+\phi_{l}\right) \\
= & 6 \pi \mu_{g} U_{g} a K\left(\phi_{s}\right) \\
& \times\left[1+\frac{\delta_{0}}{a}\left\{1+\frac{\phi_{l} a}{\delta_{0}} \frac{K^{\prime}}{K}\right\}+O\left(\delta_{0} / a\right)^{2}\right],
\end{aligned}
$$

where $K^{\prime}=d K / d \phi_{s}$ and $\phi_{l}$ is the liquid phase volume fraction given by

$$
\phi_{l}=n \int \delta d A=2 \pi n a^{2} \int_{0}^{\pi} \delta \sin \theta d \theta=3.88 \phi_{s}\left(\delta_{0} / a\right) .
$$

The coefficient 3.88 in the above expression corresponds to the case when all the liquid arrives at the north pole of particle, i.e., when $\theta_{0}=0$. The error in using the above expression is $O\left(\theta_{0}^{4 / 3}\right)$ for small but nonzero $\theta_{0}$. Combining (37) with (36), and using the definition of $f_{1}$ [cf. (34)], an approximate expression for $f_{1}$ is obtained as given by

$$
f_{1}=1+3.88 \phi_{s} K^{\prime} / K \text {. }
$$

The first term on the right-hand side of the above expression represents the effect of liquid film increasing the apparent

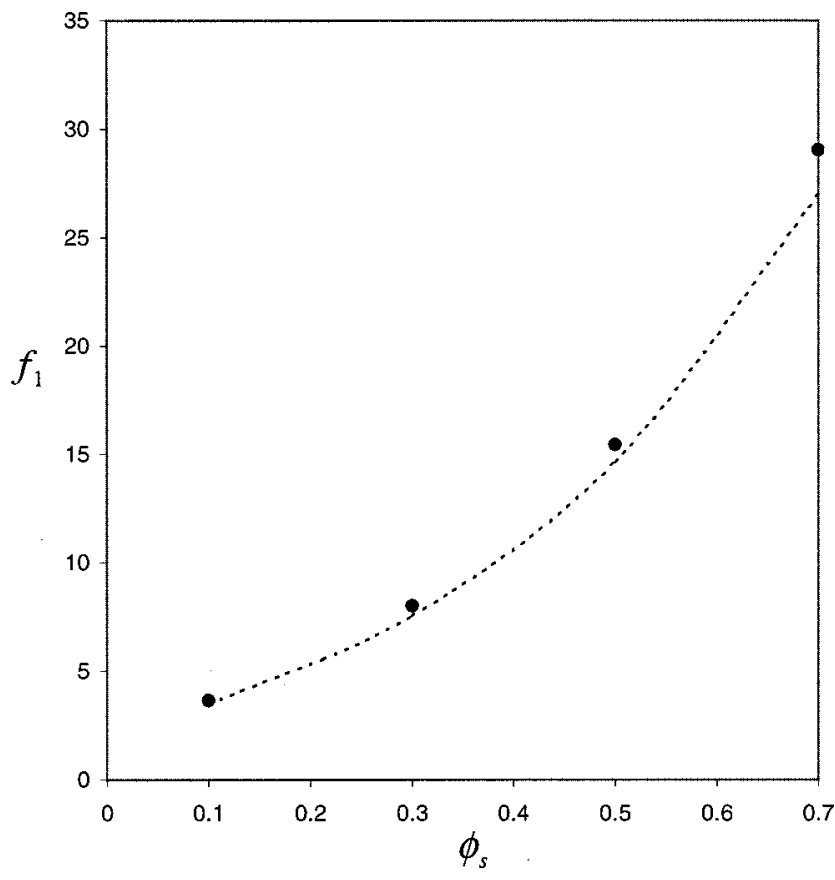

FIG. 2. The coefficient $f_{1}$ as function of $\phi_{s}$ for the face-centered cubic array of spheres. The filled circle represents the exact results and the dashed line the approximate relation given by (38).

size of the particle while the second term represents the effect of decrease in the pore space volume fraction for the gas flow. The results of numerical computations for $f_{1}$ for the face-centered cubic array are compared against the above approximate estimate for $f_{1}$ in Fig. 2. For small volume fractions, $K^{\prime}$ was evaluated analytically by differentiating the small $\phi_{s}$ expansion for $K$ given by Sangani and Acrivos ${ }^{5}$ while numerical differentiation using a central difference formula was used for $\phi_{s}>0.3$. We see that the numerical results for $f_{1}$ are in excellent agreement with the simple expression (38).

The above approximate theory assumed that the film thickness is uniform and equal to $\delta_{0}$ while the film thickness used in computing $f_{1}$ was given by $\delta=\delta_{0}(\sin \theta)^{-2 / 3}$. To check the accuracy of the numerical results, we have also determined $f_{1}$ for the case of a uniform film $\delta=\delta_{0}$ for which $\phi_{l}=3 \phi_{s}\left(\delta_{0} / a\right)$ and $f_{1}$ is given by

$$
f_{1}=1+3 \phi_{s} K^{\prime} / K \text {. }
$$

The above result is exact for periodic arrays. Table II shows $f_{1}$ as a function of $\phi_{s}$ for the face-centered cubic arrays with

TABLE II. Comparison between approximate [Eqs. (38)-(39)] and exact (computed) values of $f_{1}$ for the two cases of liquid film thickness distribution at various $\phi_{s}$ for the face-centered cubic array.

\begin{tabular}{cccccc}
\hline \hline & \multicolumn{3}{c}{$\delta=\delta_{0}$} & & \multicolumn{2}{c}{$\delta=\delta_{0}(\sin \theta)^{-2 / 3}$} \\
\cline { 2 - 3 } \cline { 5 - 6 }$\phi_{s}$ & Eq. (39) & Exact & & Eq. (38) & Exact \\
\hline 0.1 & 3.06 & 3.06 & & 3.66 & 3.47 \\
0.3 & 6.42 & 6.43 & & 8.02 & 7.56 \\
0.5 & 12.18 & 12.16 & & 15.44 & 14.67 \\
0.7 & 22.64 & 22.69 & & 29.05 & 26.99 \\
\hline \hline
\end{tabular}




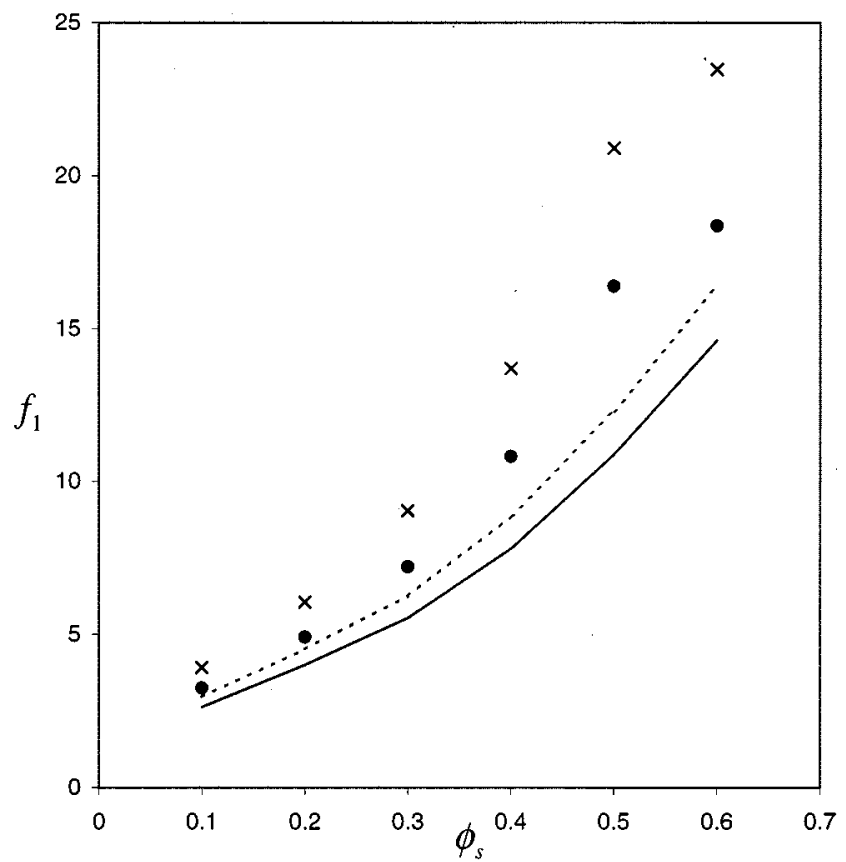

FIG. 3. The coefficient $f_{1}$ as a function of $\phi_{s}$ for the random arrays of spheres. The filled circles and solid line correspond, respectively, to the exact results and the approximate relation for the case of $\delta=\delta_{0}$ at low gas flow rates. The crosses and the dashed lines are the corresponding results for the distribution $\delta=\delta_{0}(\sin \theta)^{-2 / 3}$.

the two liquid film thickness distributions. These are compared with (38) and (39). We see that indeed there is an excellent agreement between (39) and the numerical results for the constant $\delta$ case. (38) slightly overpredicts $f_{1}$ for the case of $\delta=\delta_{0}(\sin \theta)^{-2 / 3}$. In fact, it appears that the numerical results for both cases are in a reasonable agreement with (39).

For random arrays the above result for the constant $\delta$ case is not exact because the spatial distribution of the particles with radius $a$ and volume fraction $\phi_{s}+\phi_{l}$ is not the same as for the random arrays with volume fraction $\phi_{s}$ but with the particle radius changed to $a+\delta_{0}$. Nevertheless it is of some interest to compare the relation (39) with the results for $f_{1}$ for random arrays. We used 20 configurations of hardsphere random arrays with 16 particles per unit cell to determine $f_{1}$ for random arrays with uniform film thickness. Numerical differentiation of $K$ for random arrays is difficult and hence we used the following fit of $K$ for random arrays to obtain estimates of $K^{\prime}:{ }^{16}$

$$
\begin{aligned}
K= & \frac{1+3\left(\phi_{s} / 2\right)^{1 / 2}+(135 / 64) \phi_{s} \ln \phi_{s}+17.14 \phi_{s}}{1+0.681 \phi_{s}-8.48 \phi_{s}^{2}+8.16 \phi_{s}^{3}} \\
& \left(\phi_{s} \leqslant 0.45\right) .
\end{aligned}
$$

Figure 3 shows results for $f_{1}$ for both the uniform and the nonuniform thickness distributions as a function of $\phi_{s}$ for random arrays. The solid line in that figure represents the approximate value of $f_{1}$ predicted by (39) with $K^{\prime}$ and $K$ evaluated using (40) for the uniform thickness distribution case.

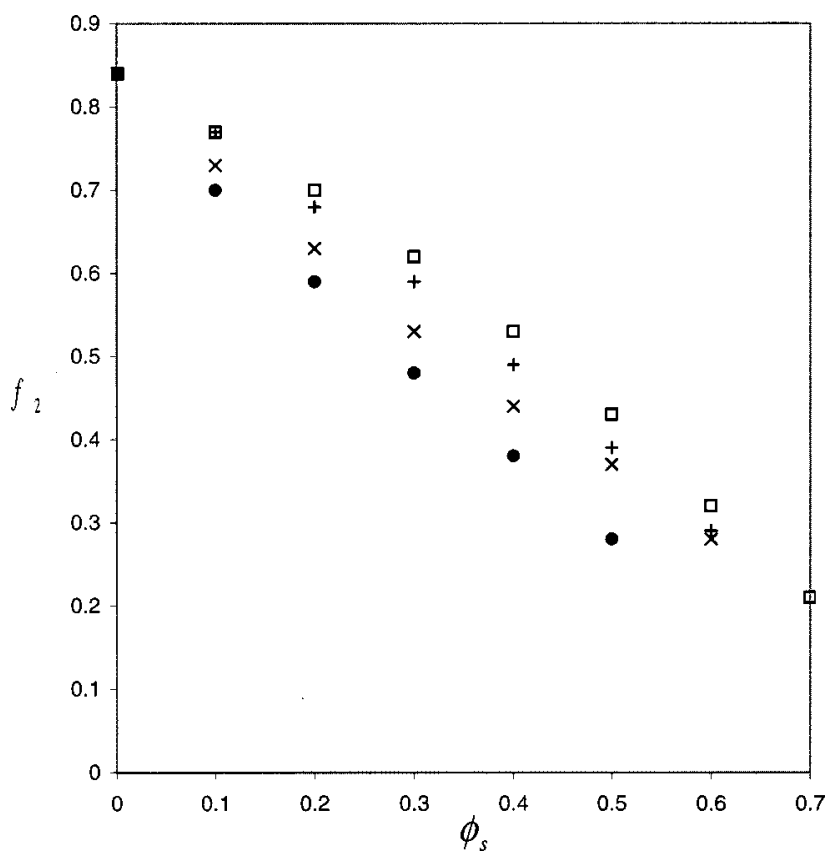

FIG. 4. The coefficient $f_{2}$ as a function of $\phi_{s}$ for periodic and random arrays of spheres. $\theta_{0}=\pi / 20$. The filled circles are the results for the simple cubic arrays, pluses for the body-centered cubic arrays, squares for the facecentered cubic arrays, and crosses for the random arrays of spheres. The solid line represents the fit $f_{1}=0.84-\phi_{s}$.

We now present the results for the effects of the motion within the liquid film, i.e., for $f_{2}$ and $f_{3}$. The results for these two quantities for the face-centered cubic array are given in Table I for two values of $\theta_{0}$ corresponding to $\theta_{0}$ $=\pi / 40$ and $\theta_{0}=\pi / 20$. Note that the results for $f_{1}$ discussed earlier corresponded to $\theta_{0}=0$, i.e., assuming that all the liquid arrives exactly at the north pole $\theta=0$. Small values of $\theta_{0}$ would have affected the results for $f_{1}$ by an insignificant amount. The same is true for $f_{2}$. In the limit of small $\phi_{s}, f_{2}$ is related to $\left(\nabla_{s} \cdot \mathbf{v}_{s}\right)_{10}$ by simply

$$
f_{2}=\left[\nabla_{s} \cdot\left(\mathbf{e}_{\theta} \sin \theta\right)\right]_{10}=\frac{2}{3} \int_{0}^{\pi} \frac{\cos \theta}{(\sin \theta)^{1 / 3}} d \theta=0.8425
$$

for $\theta_{0}=0$. Here, $\left(\nabla_{s} \cdot \mathbf{v}_{s}\right)_{10}$ is the coefficient of $Y_{10}$ in the spherical harmonic expansion of $\nabla \cdot \mathbf{v}_{s}$ [cf. (27)]. The correction to the above for small but finite $\theta_{0}$ can be shown to be small, of $O\left(\theta_{0}^{14 / 3}\right)$. Thus, $f_{2}$ is essentially independent of $\theta_{0}$ as long as the latter is not too large. The results for $f_{2}$ for all the three cubic arrays and periodic arrays with $\theta_{0}=\pi / 20$ are shown in Fig. 4. The solid line in that figure represents the approximate relation

$$
f_{2}=0.84-\phi_{s} .
$$

Finally, we note that the effect of liquid film distribution near the north and south poles is the most significant for $f_{3}$, the results for which for the face-centered cubic array were given in Table I. Similar strong dependence on $\theta_{0}$ is expected for the other arrays. 


\section{THE LOADING REGIME}

We now consider gas velocities for which the traction exerted by the gas at the gas-liquid interface is sufficiently large to affect the liquid flow and the liquid film thickness, i.e., we consider $U_{g}=O\left(U_{g l}\right)$ with $U_{g l}$ defined by (1). The ratio of the characteristic gas and liquid velocities in this regime is $O\left(U_{g l} / A\right)$, or $O\left(\mu_{l} a /\left(\mu_{g} \delta_{0}\right)\right)$, which is typically very large. Thus the pressure drop contribution due to upward moving average gas velocity is much greater than the downward moving liquid motion. In other words, the main effect of liquid flow on the gas pressure drop is through the finite thickness of the film and not the nonzero velocity at the gas-liquid interface. Note also that $f_{2}$ was generally much smaller than $f_{1}$. Thus we may set $A=0$ in determining the effect of liquid film on the gas pressure drop and liquid holdup. In other word, we must solve for the liquid flow and film thickness allowing for the effect of gas flow but that in determining the gas flow we may use the no-slip boundary condition at the gas-liquid interface. We shall begin with the simple capillary model of packed beds. The results obtained using this model will be compared with those to be obtained later for fixed beds.

\section{A. The capillary model}

In the simplest model of a packed bed/porous medium, the medium is assumed to consist of equal-size, straight capillaries of radius $a_{c}$ oriented in the direction parallel to the mean flow. The radius $a_{c}$, the number of capillaries per unit cross-section, and the average gas velocity $U_{g c}$ through the capillaries are chosen such that the porosity, the superficial velocity through the medium, and the pressure gradient in the gas for a dry bed are the same as in the actual medium. For example,

$$
\begin{aligned}
& U_{g c}=U_{g} /\left(1-\phi_{s}\right), \\
& \frac{a^{2}}{a_{c}^{2}}=(9 / 16) \phi_{s}\left(1-\phi_{s}\right) K\left(\phi_{s}\right) .
\end{aligned}
$$

A note on the notation used in this section will be helpful to the reader. The subscript $c$ is used to denote quantities concerning the capillary model; the subscript 0 is used to denote a low gas flow rate limit quantity; the gas and liquid flows will be characterized by subscripts $g$ and $l$, respectively; the solid volume fraction will be denoted by $\phi_{s}$, and the critical gas flow rate conditions to be introduced later in this section will be denoted by the subscript crit.

For random fixed beds of spherical particles with $\phi_{s}$ in the range of $0.5-0.7$, the dry bed force coefficient $K\left(\phi_{s}\right)$ can be estimated from the experimentally determined Carman correlation

$$
K=\frac{10 \phi_{s}}{\left(1-\phi_{s}\right)^{3}},
$$

whereas (40) may be used for estimating $K$, and hence $a / a_{c}$ for beds with $\phi \leqslant 0.45$. For periodic arrays, one may use the results for $K$ reported by Zick and Homsy ${ }^{4}$ and Sangani and Acrivos. $^{5}$
Let us now consider the gas-liquid flow through such capillaries. We assume that the liquid flows down along the inner walls of capillaries with a uniform film of thickness $\delta_{c}$ while the gas moves upward through the central core, $0 \leqslant r$ $\leqslant a_{c}-\delta_{c}$. Both the gas and liquid velocities are assumed to be unidirectional and functions only of radial position. We shall present here an approximate analysis valid for the case when $\delta_{c}$ is small compared with $a_{c}$ and when the ratios of viscosities and densities $\mu_{g} / \mu_{l}$ and $\rho_{g} / \rho_{l}$ are much smaller than unity. Appendix B gives the results obtained by an exact analysis in which these approximations are not made. The predictions from the two analyses will be compared later in the section.

Since the liquid velocity is much smaller than the gas velocity, the gas velocity can be taken to be zero at $r=a_{c}$ $-\delta_{c}$. The pressure gradient in the gas is then related to $U_{g c}$ by

$$
|\nabla p|=\frac{8 \mu_{g} U_{g c}}{a_{c}^{2}\left(1-\epsilon_{c}\right)^{4}},
$$

where $\epsilon_{c}=\delta_{c} / a_{c}$ is the nondimensional film thickness. The downward flow of liquid due to gravity equals $\left(2 \pi \rho_{l} g a_{c}^{4} / 3 \mu_{l}\right) \epsilon_{c}^{3}$ when $\epsilon_{c} \ll 1$ while that due to upward moving gas is $\left(4 \pi a_{c}^{2} \mu_{g} U_{g c} \epsilon_{c}^{2}\right) /\left(\mu_{l}\left(1-\epsilon_{c}\right)^{3}\right), U_{g c}$ being the superficial gas velocity through the capillary. The difference between the two gives the total volumetric liquid flow rate through a capillary. This gives the relation between the nondimensional gas velocity and the liquid film thickness as given by

$$
\epsilon_{c}^{3}-6 U_{c}^{*} \epsilon_{0 c} \frac{\epsilon_{c}^{2}}{\left(1-\epsilon_{c}\right)^{3}}=\epsilon_{0 c}^{3},
$$

where

$$
\begin{aligned}
U_{c}^{*} & =\frac{U_{g c} a^{2}}{U_{g l, c} a_{c}^{2}}, \\
\epsilon_{0 c}^{3} & =\frac{3 \mu_{l} U_{l}}{2\left(1-\phi_{s}\right) a_{c}^{2} \rho_{l} g},
\end{aligned}
$$

$U_{l}$ being the superficial velocity of the liquid through the medium. Note that $\epsilon_{0 c}=\delta_{0 c} / a_{c}$ is the nondimensional film thickness in the absence of gas flow. The loading velocity $U_{g l, c}$ is based on $\delta_{0 c}$ in lieu of $\delta_{0}$ used in (1). The nondimensional pressure gradient can be expressed in terms of $U_{c}^{*}$ by combining (46) and (48),

$$
\left|\nabla p^{*}\right| \equiv \frac{|\nabla p|}{\rho_{l} g}=\frac{8 \epsilon_{0 c} U_{c}^{*}}{\left(1-\epsilon_{c}\right)^{4}} .
$$

Figures 5 and 6 show the nondimensional film thickness and the pressure drop as functions of $U_{c}^{*}$ for $\epsilon_{0 c}=0.02 \mathrm{ob}-$ tained by the approximate expressions given here and the exact expressions given in Appendix B. The latter requires ratios of gas to liquid viscosities and densities. We used $\mu_{g} / \mu_{l}=0.02$ and $\rho_{g} / \rho_{l}=0$. We see that the predictions of the two models are essentially the same as long as $\epsilon_{c}$ is less than about 0.1 . For larger film thicknesses the exact solution 


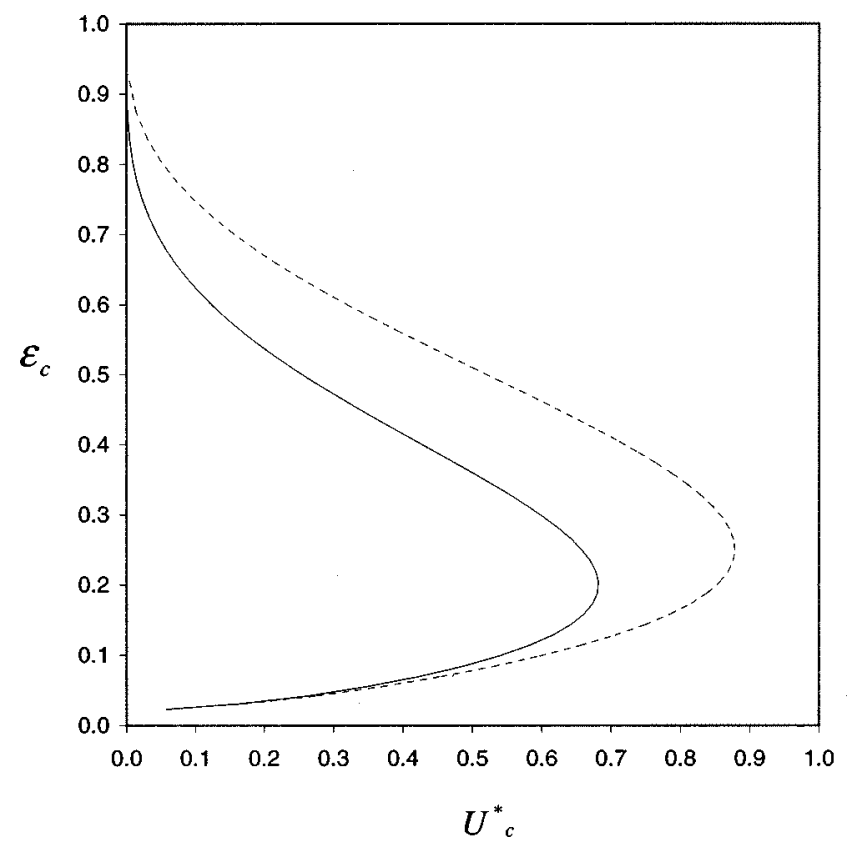

FIG. 5. $\boldsymbol{\epsilon}_{c}$ vs $U_{c}^{*}$. The solid line represents the exact result and dashed line the approximate.

given in Appendix B is necessary to provide accurate estimates of pressure drops. We also see the existence of two steady states for most values of $U_{c}^{*}$. These two solution branches meet at the turning point $U_{c}^{*}=U_{\text {crit }, c}^{*}$. No steady solutions exist for $U_{c}^{*}>U_{\text {crit }, c}^{*}$. The lack of steady solution at such high gas flow rates is interpreted in the literature to be related to the onset of flooding. For example, Dankworth and Sundaresan ${ }^{10}$ analyzed averaged-equations for gas and liquid flows through packed beds. Although different from the capillary model, their analysis also showed qualitatively the

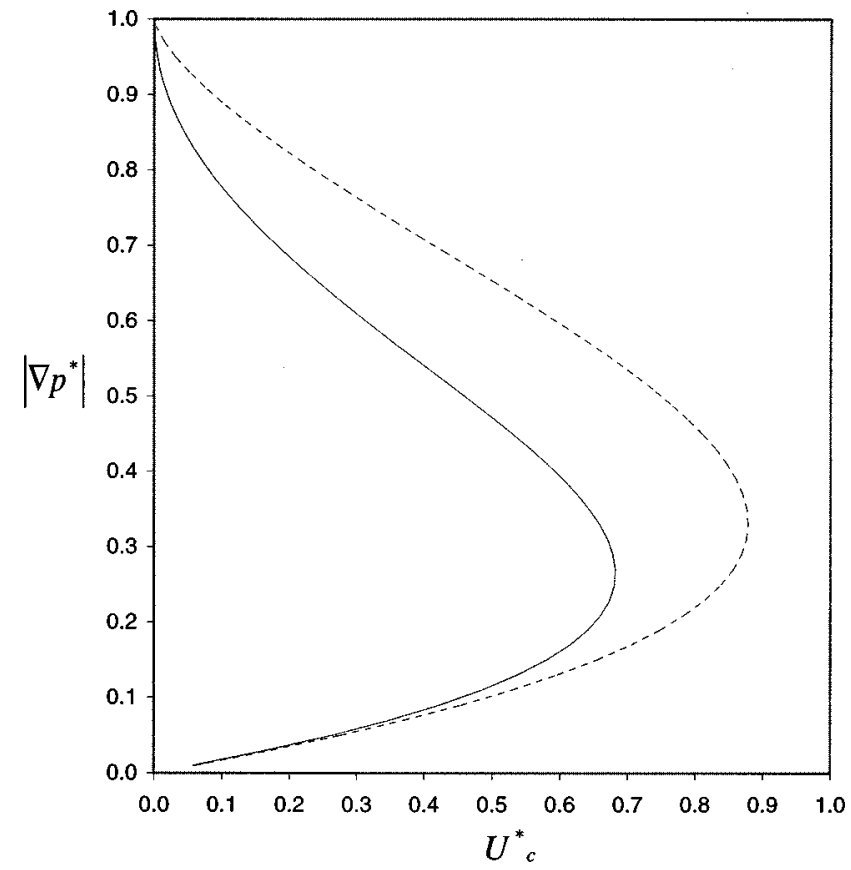

FIG. 6. $\left|\nabla p^{*}\right|$ vs $U_{c}^{*}$. The solid line represents the exact result and dashed line the approximate.

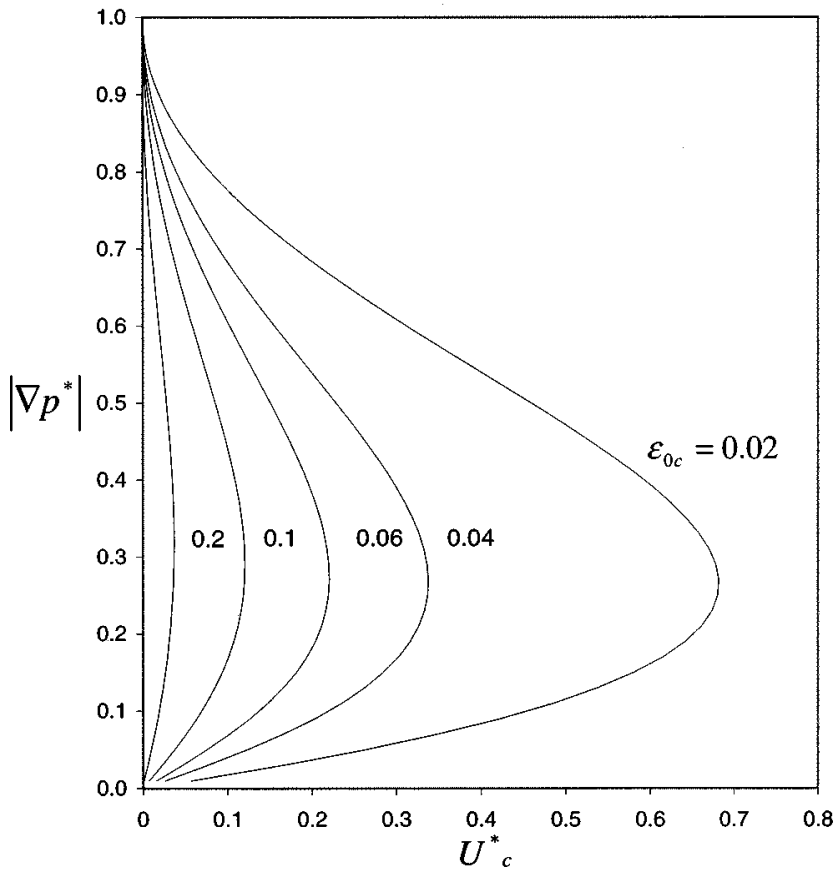

FIG. 7. The nondimensional pressure gradient $\left|\nabla p^{*}\right|$ vs $U_{c}^{*}$ at various $\epsilon_{0 c}$.

same behavior. The turning point was interpreted by these investigators as corresponding to the flooding point.

Figure 7 shows the pressure gradient as a function of $U_{c}^{*}$ for several different values of $\epsilon_{0 c}$ using the exact solution given in Appendix B. The behavior is qualitatively the same at all the indicated values of $\epsilon_{0 c}$. It is interesting to note that the nondimensional pressure at the turning point is approximately constant, at about 0.25 as $\epsilon_{0 c}$ is varied from 0.02 to 0.2 .

Figure 8 shows $U_{\text {crit,c }}^{*}$ as a function of $\epsilon_{0 c}$. The solid line in that figure corresponds to an approximate fit

$$
U_{\text {crit }, c}^{*} \epsilon_{0 c}=0.013 \text {. }
$$

The uniform thickness flow of liquid down a vertical wall is generally unstable unless it is stabilized by sufficiently large surface tension. The upper branch in Figs. 5-7 is very unstable so that in practice the pressure drop and liquid film thickness are expected to correspond to the lower branch. Dankworth and Sundaresan ${ }^{10}$ have performed linear stability analysis of the steady solutions obtained from the averaged equations for gas and liquid flows and also found that the upper branch is very unstable. The lower branch stability depended strongly on the surface tension, and, in particular, for the case of zero interfacial tension, the lower branch was found to be unstable at all gas flow rates. It may be noted, however, that there is no experimental evidence to indicate that the lower branch is unstable for gas-liquid flows through packed beds.

\section{B. Fixed bed of particles}

We now consider the loading regime for a fixed bed of particles. In this regime the gas flow affects the liquid film thickness distribution on the surface of the particles and we 


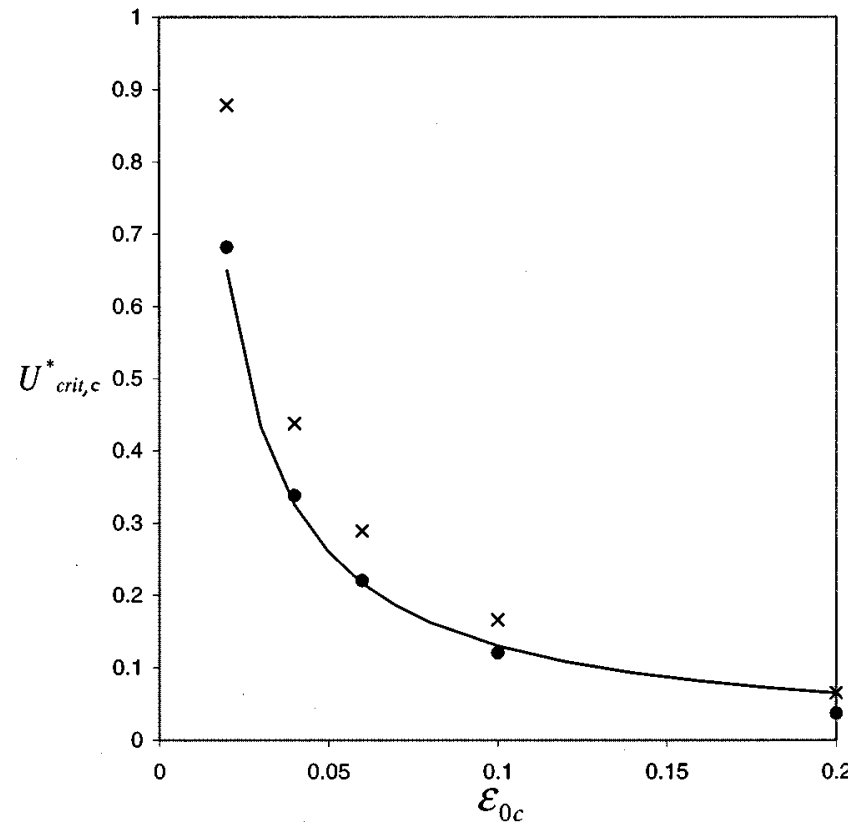

FIG. 8. $U_{\text {crit }, c}^{*}$ vs $\epsilon_{0 c}$. The filled circles represent the exact results and crosses the approximate. The solid line corresponds to the fit $U_{\text {crit }, c}^{*} \epsilon_{0 c}$ $=0.013$.

must use a numerical method to determine this distribution. We shall use both steady and unsteady flow equations to determine the film distribution.

Let us denote by $\mathbf{q}_{s}$ the surface flow at a point $(\theta, \varphi)$ on the surface of a particle,

$$
\mathbf{q}_{s}(\theta, \varphi)=\int_{a}^{a+\delta} \mathbf{u}_{s}(r, \theta, \varphi) d r,
$$

where $\delta$ is the film thickness at $(\theta, \varphi)$ and $\mathbf{u}_{s}$ is the velocity parallel to the surface of the particle. The mass balance for the liquid gives

$$
\frac{\partial \delta}{\partial t}+\nabla_{s} \cdot \mathbf{q}_{s}=S
$$

where $S$ is the source. $S$ is zero everywhere except at $\theta=0$ and $\pi$ for the case when the fluid arrives at the north pole, flows down the surface, and leaves from the south pole of the particle. For the case of the uniform thickness model $\delta$ $=\delta_{0}$, we take

$$
S=\frac{2 \rho_{l} g}{3 \mu_{l}} \delta_{0}^{3} \cos \theta,
$$

so that $\delta=\delta_{0}$ is a steady state solution of (53) when the tangential stress at the gas-liquid interface is zero.

Since the Reynolds number for the liquid flow is much smaller than for the gas flow, and the gas Reynolds number is assumed to be small compared with unity, we shall neglect the inertial terms in the momentum equation for the liquid. The surface flow is then given by

$$
\mathbf{q}_{s}=\frac{\rho_{l} g}{3 \mu_{l}}\left(\mathbf{g}_{s}-r^{-1} \nabla_{s} p_{l}\right) \delta^{3}-\frac{\mathbf{f}_{s}}{2 \mu_{l}} \delta^{2},
$$

where $\mathbf{g}_{s}=g \sin \theta \mathbf{e}_{\theta}, p_{l}$ is the pressure in the liquid film, and $\mathbf{f}_{s}$ is the tangential stress at the gas-liquid interface. Note that $\mathbf{f}_{s}$ is a function of $\theta$ and $\varphi$. The gas and liquid flows are coupled through this stress.

As in the case of low gas flow rates analysis we write the gas velocity as $\mathbf{u}_{g}=\mathbf{u}^{(0)}+\mathbf{u}^{(1)}$ with the boundary conditions,

$$
\mathbf{u}^{(0)}=\mathbf{0}, \quad \mathbf{u}^{(1)}=\mathbf{v} \equiv-\delta(\theta, \varphi) \frac{\partial \mathbf{u}^{(0)}}{\partial r} \quad \text { at } r=a .
$$

Note that $\mathbf{u}^{(0)}$ corresponds to the gas flow in a dry bed while $\mathbf{u}^{(1)}$ is the correction due to finite film thickness.

The numerical scheme for solving the gas and liquid flows consists of following steps: (i) The surface of a sphere is discretized into a number of triangular elements and the initial value of $\delta$ at these points is taken to be the same as corresponding to the low gas flow analysis. (ii) With $\mathbf{u}^{(0)}$ determined a priori, the right-hand side of the second equation in (56), i.e., $\mathbf{v}$, is evaluated at the nodes of the triangular elements. The components of this velocity are expanded in spherical surface harmonics as in the low gas flow rate analysis [cf. (26)-(27)] and these expansions are used for determining $\mathbf{u}^{(1)}$. (iii) The tangential stress $\mathbf{f}_{s}$ and $\nabla_{s} p_{l}$ at $r=a$ $+\delta$ are evaluated next at all the nodes using the combined velocity field $\mathbf{u}^{(0)}+\mathbf{u}^{(1)}$. Since the surface tension is taken to be zero, $p_{l}$ is the same as the gas pressure. The surface flow $\mathbf{q}_{s}$ is evaluated at all the node points using (55). (iv) Next, $\nabla_{s} \cdot \mathbf{q}_{s}$ is evaluated at the node points using a second-order difference formula. Since the liquid flow is primarily in the $\theta$-direction we use a backward difference formula for the derivative with respect to $\theta$ and a central difference formula for the derivative with respect to $\varphi$, i.e., we evaluate $\nabla_{s} \cdot \mathbf{q}_{s}$ using

$$
\begin{aligned}
\left(\sin \theta \nabla \cdot \mathbf{q}_{s}\right)_{i, j}= & (1 / 2 \Delta \theta)\left[3\left(q_{\theta} \sin \theta\right)_{i, j}\right. \\
& \left.-4\left(q_{\theta} \sin \theta\right)_{i-1, j}+\left(q_{\theta} \sin \theta\right)_{i-2, j}\right] \\
& +(1 / 2 \triangle \varphi)\left[\left(q_{\varphi}\right)_{i, j+1}-\left(q_{\varphi}\right)_{i, j-1}\right],
\end{aligned}
$$

where $q_{\theta}$ and $q_{\varphi}$ are the components of $\mathbf{q}_{s}$, i.e., $\mathbf{q}_{s}=\mathbf{e}_{\theta} q_{\theta}$ $+\mathbf{e}_{\varphi} q_{\varphi}$, and the subscripts $i$ and $j$ correspond to a node $\left(\theta_{i}, \varphi_{j}\right)$ on the surface of the sphere. The low gas flow rate behavior is assumed to hold near the north pole, i.e., we assume that at $\theta=0$ and $\theta=\Delta \theta$, the discretization interval for $\theta, \delta=\delta_{0}(\sin \theta)^{-2 / 3}$ at all times. $\partial \delta / \partial t$ at the node points are evaluated next by substituting for $\nabla_{s} \cdot \mathbf{q}_{s}$ into (55). A Runge-Kutta method is used to determine $\delta(t+\triangle t), \triangle t$ being the time increment. (v) Steps (ii)-(iv) are repeated until the steady state is reached. The gas flow rate is subsequently incremented by a small amount and the steps (ii)-(v) repeated to determine the film thickness distribution, liquid holdup, and pressure drop as function of the gas flow rate for selected values of $\phi_{s}$ and $\delta_{0}$.

In an alternative method, the steady state liquid holdup and gas pressure drop are determined directly as described below. As in the transient method, the gas velocity, and hence $\mathbf{f}_{s}$ and $\nabla_{s} p_{l}$ are determined first for an assumed film thickness distribution. These quantities are used for determining $\left(q_{\theta} \sin \theta\right)_{i-1, j}, \quad\left(q_{\theta} \sin \theta\right)_{i-2, j}, \quad\left(q_{\varphi}\right)_{i, j-1}, \quad$ and $\left(q_{\varphi}\right)_{i, j+1}$ using (55). Next, $\left(\sin \theta q_{\theta}\right)_{i, j}$ is calculated using (57) 


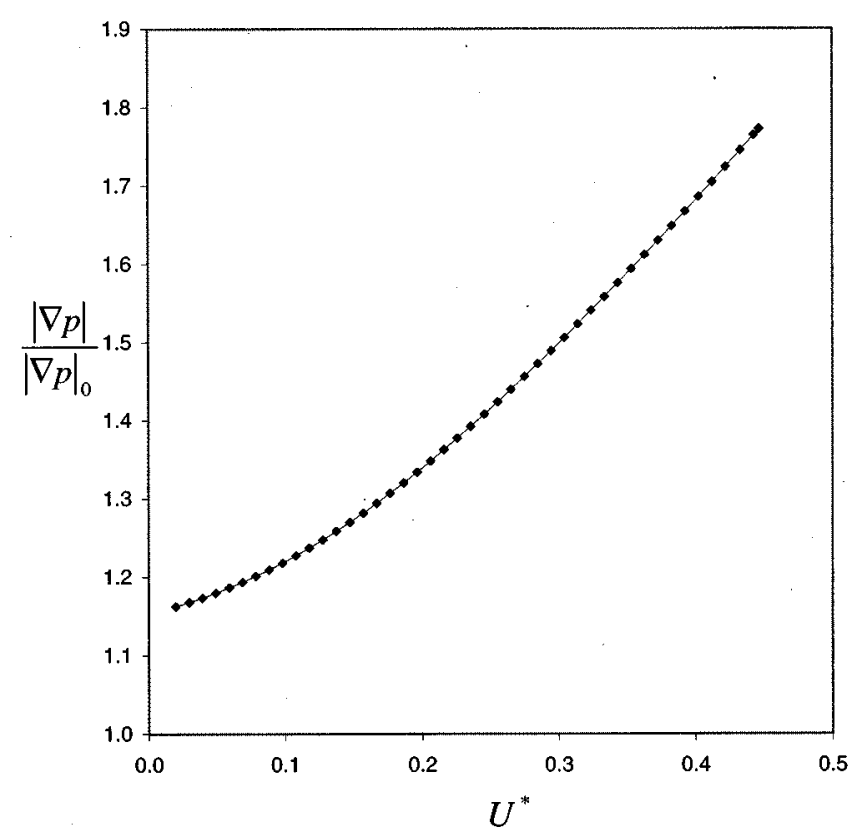

FIG. 9. The ratio of pressure gradients in wet and dry beds, $|\nabla p| /|\nabla p|_{0}$, as a function of scaled gas velocity $U^{*}$. The dots represent the results obtained by the steady state method and the line represents the results by the transient method. $\phi=0.4 ; \delta_{0} / a=0.02$; simple cubic array.

and $\nabla_{s} \cdot \mathbf{q}_{s}=0$. This is next substituted in (55) and the resulting cubic equation is solved to determine a new estimate of $\delta_{i, j}$. The cubic equation gives either three real roots or one real root. In the case of three real roots it is found that two are very close to zero while the third is positive and comparable to $\delta_{0}$. We use this third root as the new estimate of $\delta_{i, j}$. The same procedure is used for higher values of $i$ and $j$ until the new estimates of $\delta_{i, j}$ are obtained at all the node points. This new distribution is used to solve again for the gas flow and to evaluate the tangential stress, etc., at the node points. The procedure is repeated until the sum of $\delta$ at all nodes converges. Most calculations to be presented here were obtained with $\triangle \theta=\pi / 40$ and $\triangle \varphi=\pi / 20$ and the sum of $\delta$ 's was required to converge to within $10^{-4}$. The calculations were started with low gas flow rates where the precise thickness distribution is known.

Figures 9 and 10 show the results of computations for $\delta_{0}=0.02$ and $\phi_{s}=0.4$ for the case of a simple cubic array of spheres. We see that the results obtained by the two methods are in excellent agreement with each other. The pressure gradient for the dry bed is denoted by $|\nabla p|_{0}$. The normalized pressure gradient, i.e., $|\nabla p| /|\nabla p|_{0}$, approaches $1+f_{1}$ as $U^{*} \rightarrow 0$. As expected both the liquid holdup and the normalized pressure gradient increase with increasing $U^{*}$. It should be noted that the gas velocity is scaled by the loading velocity, i.e., $U^{*}=U / U_{g l}$ with $U_{g l}$ given by (1). The low gas flow rate holdup was determined using $\phi_{l 0}=3.88 \phi_{s}\left(\delta_{0} / a\right)$ while $\phi_{l}$ was obtained by integrating $\delta$ over the surface of the sphere [cf. the first equality in (37)].

Figure 11 shows the film thickness averaged over the azimuthal angle $\varphi,\langle\delta(\theta)\rangle_{\varphi}$, for selected gas flow rates. The film thickness is symmetric around $\theta=90^{\circ}$. At low gas flow rates the film thickness decreases monotonically as $\theta$ is var-

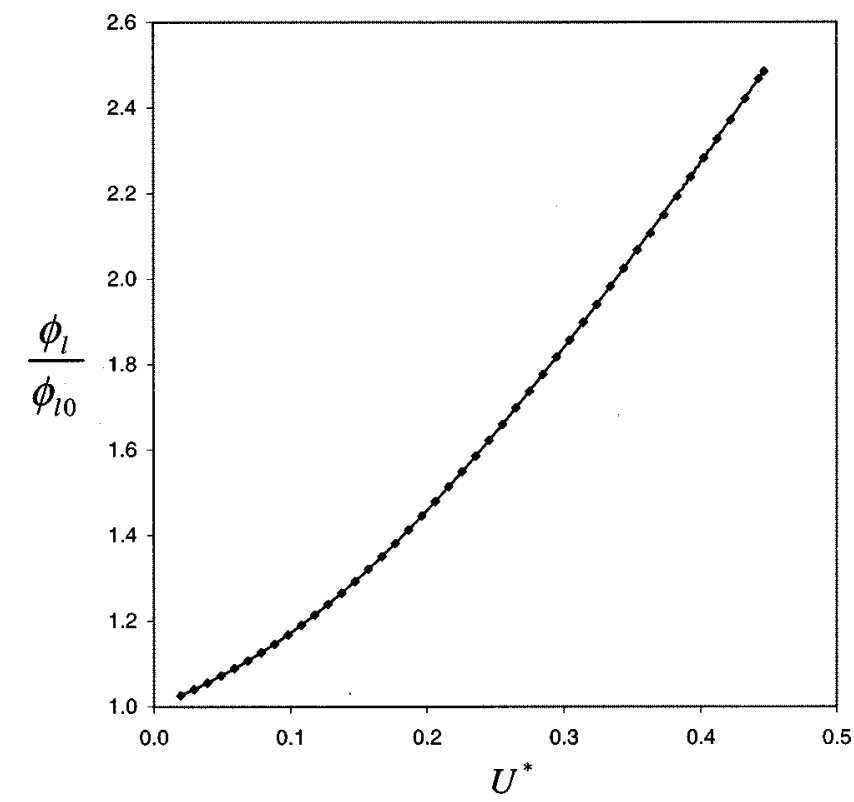

FIG. 10. $\phi_{l} / \phi_{l 0}$ vs $U^{*}$. The dots are the result by the steady state method and the line the transient method. $\phi=0.4 ; \delta_{0} / a=0.02$; simple cubic array.

ied from 0 to $90^{\circ}$. As the gas flow rate is increased, the film thickness near $\theta=90^{\circ}$, where the traction exerted by the gas is a maximum, increases. Figure 12 shows the variation in $\delta$ with the azimuthal angle $\varphi$ at $\theta=90^{\circ}$. The maximum film thickness occurs at $\varphi=45^{\circ}$.

At $U^{*} \simeq 0.45$ the liquid films on the surface of the two adjacent spheres overlap at $\theta=90^{\circ}$. Our numerical scheme for computing gas flow is not valid when the films overlap and hence we have not computed the pressure drop and holdup (Figs. 9 and 10) beyond this gas flow rate.

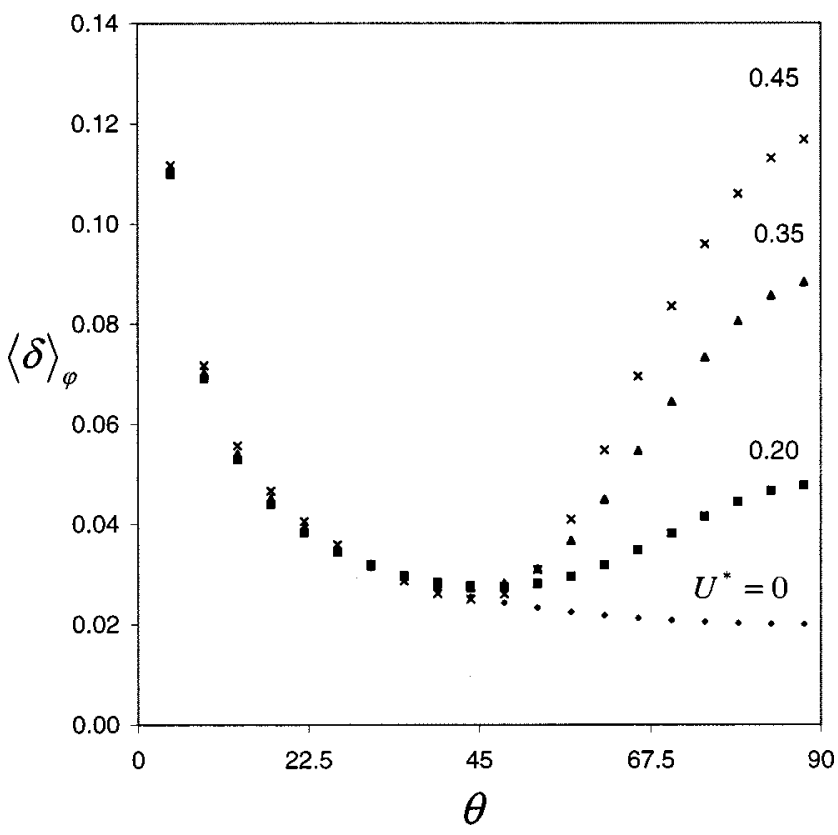

FIG. 11. The azimuthal angle-averaged film thickness, $\langle\delta\rangle_{\varphi}$, as a function of $\theta$ at various $U^{*}$. 


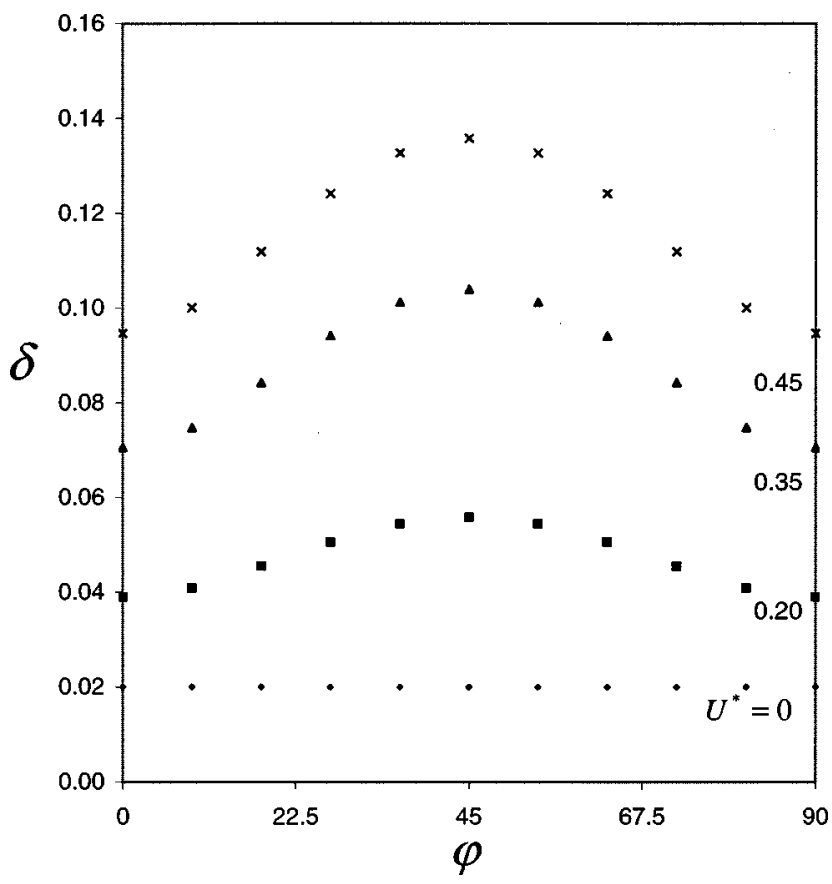

FIG. 12. The thickness of liquid film at $\theta \simeq 90^{\circ}$ as a function of $\varphi$ at various $U^{*}$ for a simple cubic array with $\phi_{s}=0.4$ and $\delta_{0} / a=0.02$.

Figures 13 and 14 show results for the case when the initial liquid film thickness is uniform over the surface of the spheres. Once again the pressure drop and holdup increase with the increasing gas flow. Figure 15 shows variations in the $\varphi$-averaged film thickness, $\langle\delta\rangle_{\varphi}$, as a function of $\theta$. We see that increasing the gas flow rate increases the thickness mostly near $\theta=90^{\circ}$ where the traction exerted by the gas is maximum. Unlike the previous case, however, we find that the liquid film thickness at some points on the sphere becomes zero at $U^{*} \simeq 0.35$. This is illustrated in Fig. 16 which

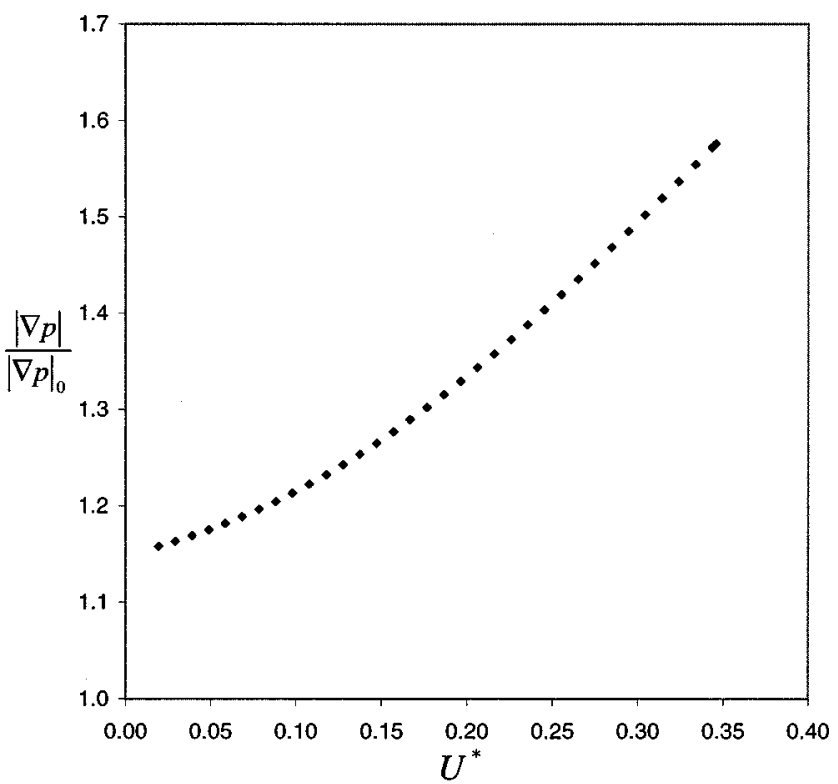

FIG. 13. $|\nabla p| /|\nabla p|_{0}$ vs $U^{*}$ for the case of uniform initial liquid film thickness. $\phi=0.4 ; \delta_{0} / a=0.02$; simple cubic array.

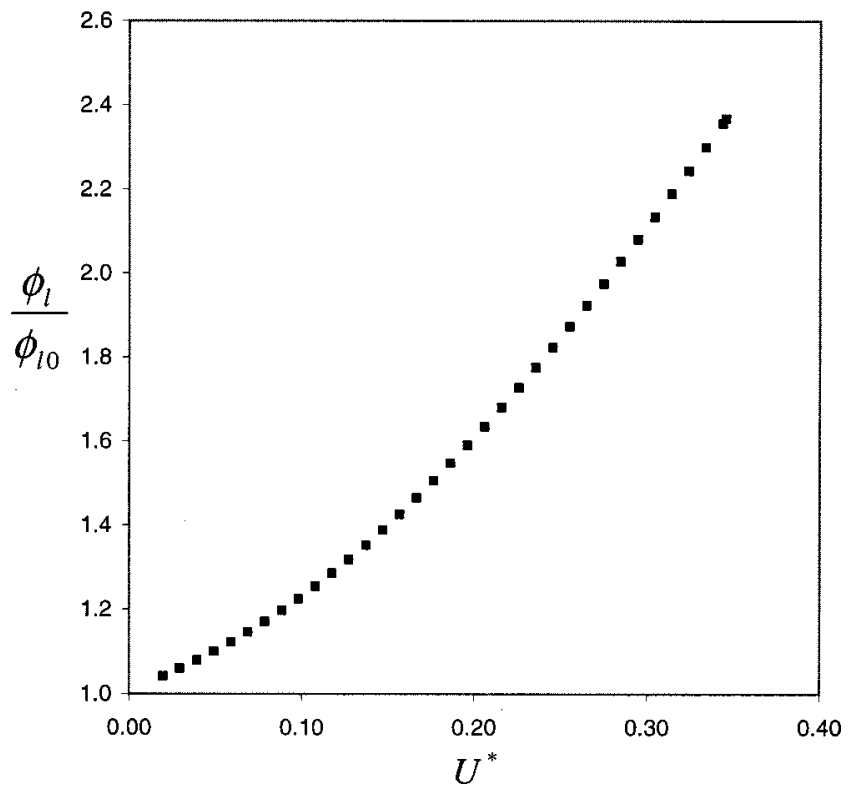

FIG. 14. $\phi_{l} / \phi_{l 0}$ vs $U^{*}$ for the case of uniform initial liquid film thickness. $\phi=0.4, \delta_{0} / a=0.02$; simple cubic array.

shows $\delta$ as a function of $U^{*}$ and $\varphi$ at $\theta=175.6^{\circ}$. We see that as $U^{*}$ is increased the film thickness begins to vary significantly with $\varphi$ exhibiting minima at $\varphi=18^{\circ}$ and at $72^{\circ}$. Very near the critical gas flow rate the symmetry around $\varphi$ $=45^{\circ}$ breaks and the film thickness at $72^{\circ}$ vanishes indicating the formation of a dry region near that point. The contact angle and other surface tension related phenomena will become important once the surface of the particle is not com-

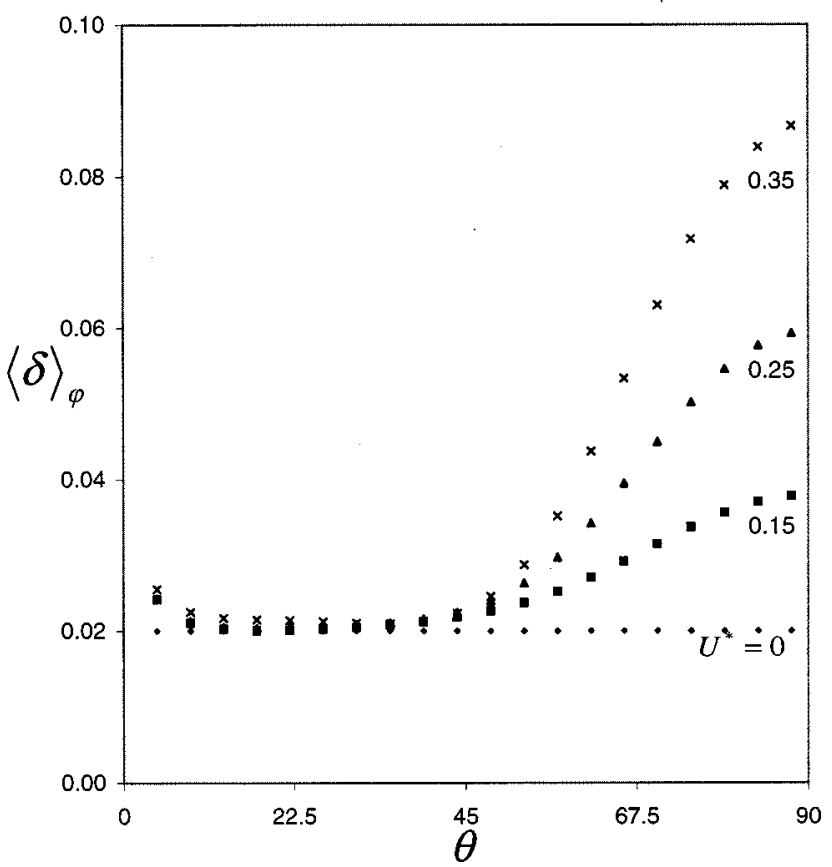

FIG. 15. The azimuthal angle-averaged film thickness, $\langle\delta\rangle_{\varphi}$, as a function of $\theta$ at various $U^{*}$ for the uniform film thickness at low gas flow rates case. $\phi_{s}=0.4 ; \delta_{0} / a=0.02 ;$ simple cubic array. 


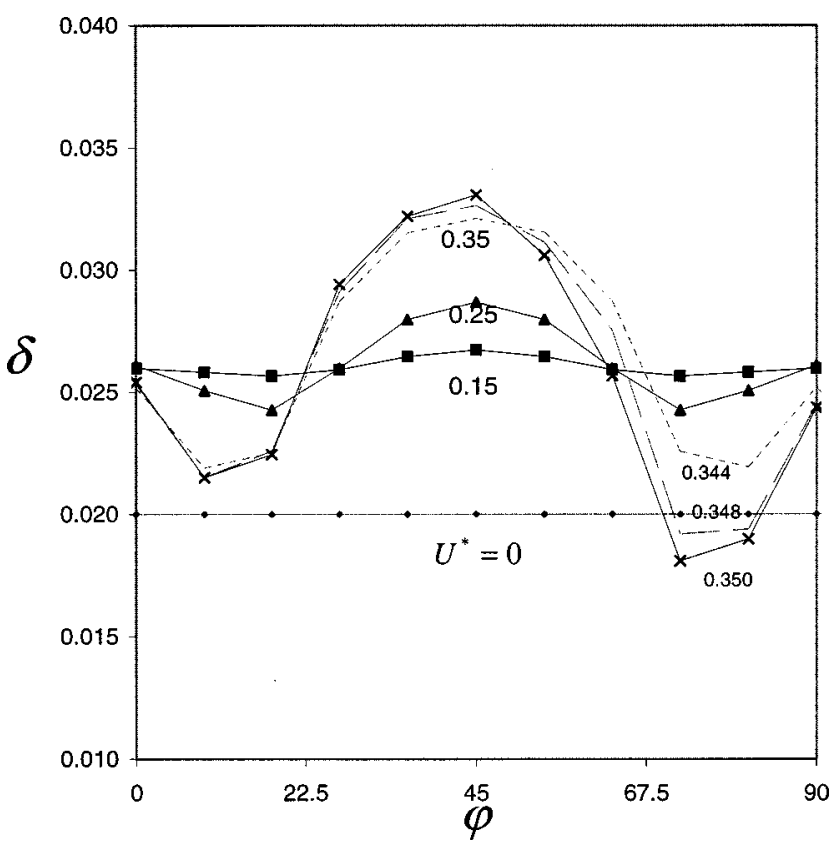

FIG. 16. $\delta$ as a function of $\varphi$ for $\theta=175.6^{\circ}$ for the case of uniform initial film thickness.

pletely wetted and this would make the calculations for higher $U^{*}$ very difficult.

Figure 17 shows the results for the body-centered cubic array with the low gas flow rate thickness driven by the gravity flow, i.e., (4). In this case the dry region occurs at much smaller gas flow rates and therefore we have been unable to compute the pressure drop and holdup at higher gas flow rates. The same applies to the face-centered cubic arrays.

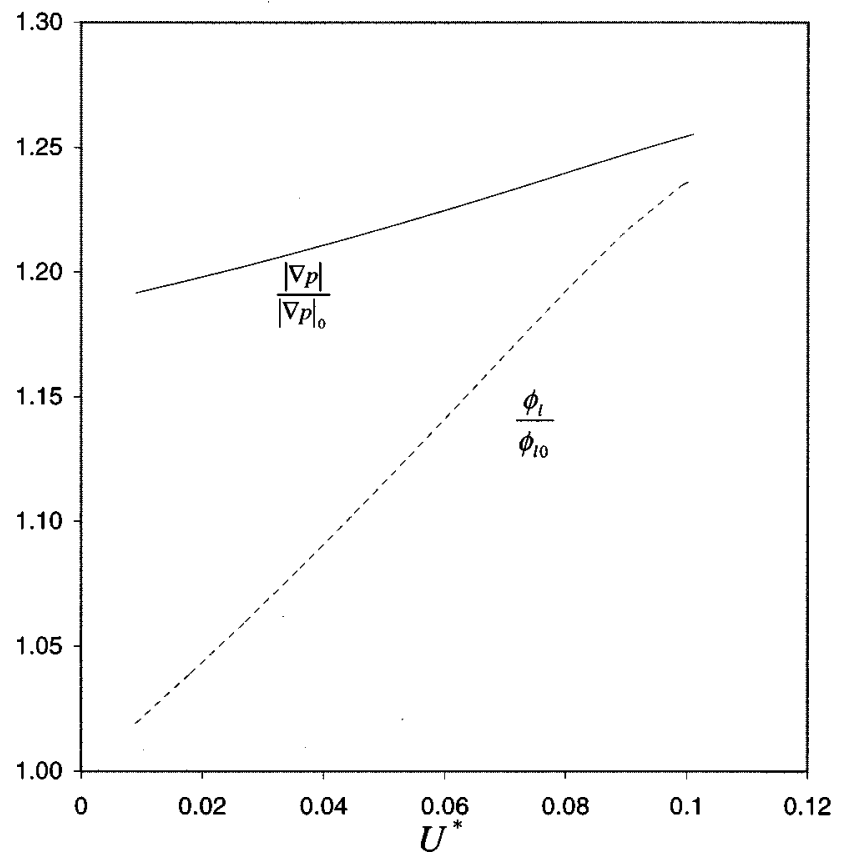

FIG. 17. $|\nabla p| /|\nabla p|_{0}$ and $\phi_{l} / \phi_{l 0}$ vs $U^{*}$ for the body-centered cubic array with $\phi=0.4$ and $\delta_{0}=0.02$.

\section{Comparison with other models}

We now compare the numerical simulation results for fixed beds with those predicted using the other models. To compare the capillary and fixed bed models we require that the liquid holdup at very low gas flow rates in the two models be the same. As mentioned earlier the radius of the capillary is chosen such that the gas pressure drop for the capillary and fixed bed models are the same in the absence of liquid flow. The results for these models will also be compared with the predictions of the averaged equations used by Dankworth and Sundaresan ${ }^{10}$ and with an approximate theory that we shall presently describe.

Dankworth and Sundaresan used the following expressions from Saez and Carbonell ${ }^{17}$ for pressure drop and holdup calculations:

$$
\begin{aligned}
& \rho_{l} g-\frac{F_{g}}{1-\phi_{s}-\phi_{l}}-\frac{F_{l}}{\phi_{l}}=0, \\
& F_{g}=\frac{45 \mu_{g} \phi_{s}^{2}\left(1-\phi_{s}\right)^{1.8} U}{a^{2}\left(1-\phi_{s}-\phi_{l}\right)^{3.8}}, \\
& F_{l}=\left(\frac{1-\phi_{s}}{\phi_{l}}\right)^{2.43} \frac{45 \mu_{l} \phi_{s}^{2} \phi_{l}^{2} U_{l}}{a^{2} \phi_{l}\left(1-\phi_{s}\right)^{3}} .
\end{aligned}
$$

In writing the above expressions we have taken the residual liquid holdup, i.e., the holdup in the absence of gas or liquid flow, to be zero and we have set the Ergun parameter, which accounts for the effect of gas inertia, in their expressions to zero. To compare the predictions from the above expressions with the ones obtained in the present study, we choose the superficial liquid velocity $U_{l}$ in such a way that the liquid holdups at zero gas flow rate calculated using the two models are the same.

In the capillary model the traction exerted by the gas at the gas-liquid interface is directly related to the total pressure gradient while in the fixed bed model the two are not directly related. The traction depends on the magnitude of the shear stress at the interface while the pressure gradient, being related to the total drag force, also depends on the magnitude of the normal force at the surface of the particles. To account for this difference we have developed an approximate theory as follows. Let us assume that the liquid film thickness distribution is similar to the initial distribution, i.e., $\delta=\delta_{*}(\sin \theta)^{-2 / 3}$. Then volumetric flow balance gives

$$
\delta_{*}^{3}+\frac{3 f_{\theta}(\sin \theta)^{-1 / 3}}{2 \rho_{l} g} \delta_{0} \delta_{*}^{2}=\delta_{0}^{3} .
$$

This equation will not hold at all $\theta$ and $\varphi$ since we have assumed a very simple form of film distribution with only one parameter, i.e., $\delta_{*}$. To satisfy the above equation in an approximate sense we integrate it over the surface of the sphere and introduce a function $\alpha\left(\phi_{s}\right)$ defined by

$$
\int f_{\theta}(\sin \theta)^{-1 / 3} d A=-6 \pi \mu_{g} a U_{g} \alpha\left(\phi_{s}\right) K\left(\phi_{s}\right) .
$$

Thus, (61) upon integrating over the sphere surface and nondimensionalizing, leads to 


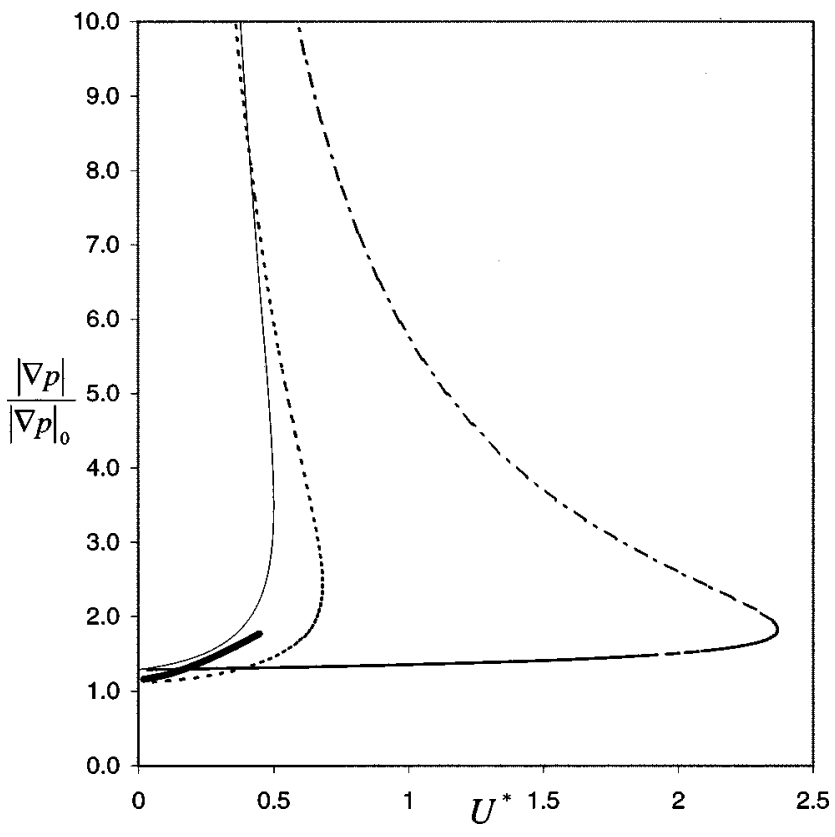

FIG. 18. A comparison among different models. The thick solid line represents the simulation, the thin solid line the approximate model, the small dashed line the capillary model, and the large dashed line the DankworthSundaresan model based on Saez and Carbonell equations.

$$
\epsilon^{3}-4 \frac{\alpha\left(\phi_{s}+\phi_{l}\right) K\left(\phi_{s}+\phi_{l}\right)}{\phi_{s} K\left(\phi_{s}\right)}(1+\epsilon) U^{*} \epsilon_{0} \epsilon^{2}=\epsilon_{0}^{3}
$$

with $\epsilon=\delta_{*} / a$ and $\epsilon_{0}=\delta_{0} / a$. Note that in writing the above equation we have accounted for the effect of finite liquid film thickness on $f_{\theta}$ by requiring that $\alpha$ and $K$ be evaluated at the total volume fraction $\phi_{s}+\phi_{l}$ and that the nondimensional radius of the particle be corrected from unity to $1+\epsilon$. The above expression is similar to the one obtained using the capillary model but with the coefficient $\alpha$ accounting now for the difference between the shear force and the total force. We have determined $\alpha$ for the simple cubic array at several $\phi_{s}$ values in the range $0-0.5$. The following expression gives a good fit to the numerical results:

$$
\alpha\left(\phi_{s}\right)=0.84\left[1+1.22 \phi_{s}+4.84 \phi_{s}^{2}\right]^{-1} .
$$

Equation (63) can be used to determine $U^{*}$ given $\epsilon$ and $\epsilon_{0}$. The normalized pressure gradient can be determined using

$$
\frac{|\nabla p|}{|\nabla p|_{0}}=\frac{K\left(\phi_{s}+\phi_{l}\right)}{K\left(\phi_{s}\right)}(1+\epsilon)
$$

with $\phi_{l}=3.88 \phi_{s} \epsilon$.

Figures 18 and 19 show a comparison among the four different methods of estimating the pressure drop and liquid holdup. The exact calculations correspond to the simple cubic array. We note that up to the point where the liquid films begin to overlap in our numerical calculations, i.e., up to $U^{*}$ of about 0.45 , the capillary model, the approximate model based on (63)-(65), and the exact method are in very good agreement with each other. The pressure drop is better predicted by the approximate model while the liquid holdup is better predicted by the capillary model. The pressure drop

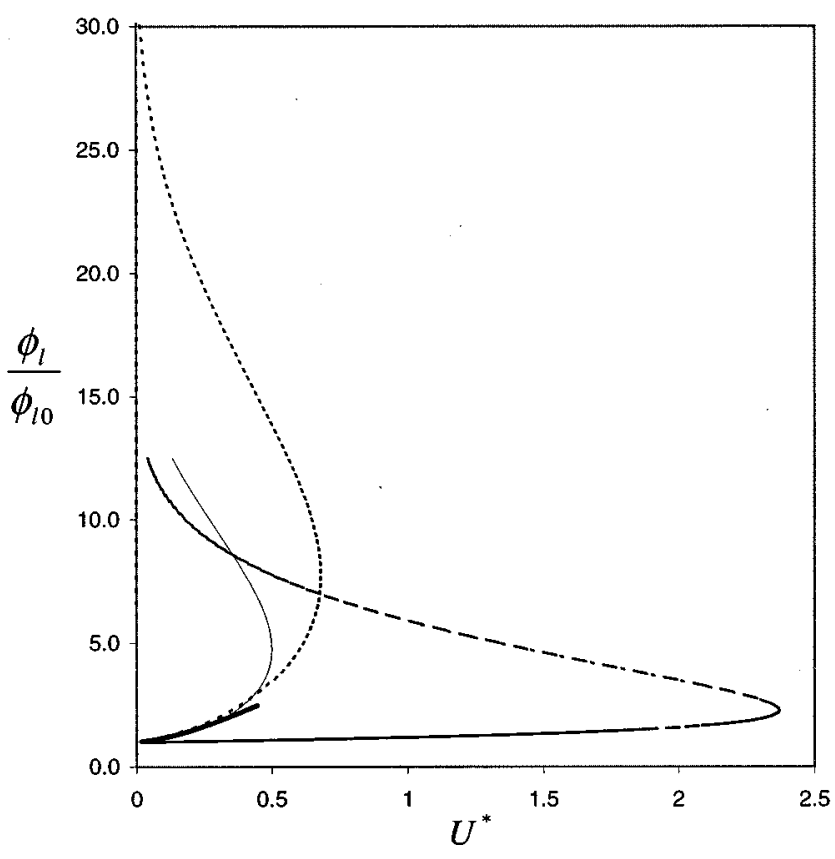

FIG. 19. A comparison among different models. The thick solid line represents the simulation, the thin solid line the approximate model, the small dashed line for the capillary model, and the large dashed line the Dankworth-Sundaresan model based on Saez and Carbonell equations.

and holdup estimated using the Saez-Carbonell (or Dankworth-Sundaresan) equations are considerably lower. Also the critical $U^{*}$ for the capillary model and the approximate model are seen to be much smaller than that predicted by the Dankworth-Sundaresan equations. This last observation may be significant since Dankworth and Sundaresan found the critical gas flow rate to be significantly greater than the flooding velocity given by the experimentally determined Sherwood correlation. ${ }^{18}$ For example, for $1.25 \mathrm{~cm}$ diam particles the flooding velocity predicted using the equations proposed by Saez and Carbonell ${ }^{17}$ was about three to four times greater than the Sherwood correlation. Dankworth and Sundaresan also carried out calculations for the flooding velocity based on equations suggested by Hutton et al. ${ }^{19}$ and found that those equations overpredicted the flooding velocity by an even greater factor. While in that comparison the gas inertia was significant, our calculations do suggest that the expressions used in the Dankworth-Sundaresan analysis tend to significantly overpredict the flooding velocity at least when the Reynolds number is small. Also, our calculations show that the simple capillary model gives reasonably accurate estimates for the pressure drop and liquid holdup. It may be noted that Specchia and Baldi ${ }^{20}$ have compared their experimental data with the Hutton et al. correlation and found the correlation to significantly underpredict the gas pressure drop in wet packed beds. This observation is consistent with our calculations.

\section{SUMMARY}

We have solved the detailed equations governing the flows of gas and liquid through fixed beds of spheres. The effect of thin liquid film on the gas pressure drop is deter- 
mined for random as well as periodic arrays of spheres. A simple analytical relation is obtained for predicting the pressure drop in the low gas flow rate regime [cf. (34), (35), and (38)]. The presence of liquid film increases the gas pressure drop by three mechanisms: an increase in the apparent size of the particles, decrease in the pore space volume fraction for the gas flow, and increase in the apparent relative velocity between the gas and the particles. Of these three, the first two effects are more significant and the equations listed above could be used to estimate their effect. At higher gas flow rates the traction produced by the gas affects the liquid holdup and makes the pressure drop-gas velocity relation nonlinear. We have been unable to carry out calculations up to high enough gas velocities to compute the flooding velocity because either of the two things happened: either the liquid formed a bridge between adjacent particles or some regions on the particle surface became dry. Both the liquid bridging and the contact line formation and their effect on the gas flow rate are difficult to incorporate in our analysis. The numerical results in the nonlinear regime are seen to be in excellent agreement with the capillary model [cf. (47) and (50)] and an approximate model [cf. (63)-(65)] developed in the present study. The equations proposed by Saez and Carbonell ${ }^{17}$ and Hutton et al. ${ }^{19}$ appear to predict lower pressure drops and liquid holdups, at least in the small Reynolds number limit. The critical gas flow rates obtained from the equations proposed by these investigators are much greater than those predicted by the capillary model and approximate model developed in the present study.

\section{ACKNOWLEDGMENTS}

Financial support for this work was provided by the National Science Foundation under Grant No. CTS-9632227. The computations were performed using the resources by the National Center for Scientific Applications at University of Illinois at Urbana-Champaign.

\section{APPENDIX A: ANALYSIS OF FLOW WITHIN THE LIQUID FILM}

Let $\quad u_{\theta}^{*}=U_{c} u_{\theta}, \quad u_{r}^{*}=U_{c} \epsilon U_{r}, \quad p^{*}=\mu_{l} U_{c} p / \delta_{0}, \quad \epsilon$ $=\delta_{0} / a$, and $r^{*}=a+\delta_{0} Y$. Here, $u_{\theta}, U_{r}$, and $p$ are scaled velocity components and pressure, $U_{c}=\rho_{l} \delta_{0}^{2} g / \mu_{l}=2 \mathrm{~A}$ is the characteristic liquid velocity, and $Y$ is the scaled distance measured from the surface of the particle. We shall determine the liquid velocity profile, the film thickness, and the gas velocity at the gas-liquid interface correct to $O(\epsilon)$ in this Appendix.

The continuity and momentum equations can be shown to reduce to

$$
\begin{aligned}
& \frac{\partial U_{r}}{\partial Y}+\frac{1}{\sin \theta} \frac{\partial}{\partial \theta}\left(u_{\theta} \sin \theta\right)+\epsilon\left\{2 U_{r}-\frac{Y}{\sin \theta} \frac{\partial}{\partial \theta}\left(u_{\theta} \sin \theta\right)\right\} \\
& \quad+O\left(\epsilon^{2}\right)=0 \\
& \frac{\partial^{2} u_{\theta}}{\partial Y^{2}}+\epsilon \sin \theta\left\{-\frac{\partial p}{\partial \theta}+2 \frac{\partial u_{\theta}}{\partial Y}\right\}+O\left(\epsilon^{2}\right)=0
\end{aligned}
$$

$\frac{\partial p}{\partial Y}=-\cos \theta+\epsilon \frac{\partial^{2} U_{r}}{\partial Y^{2}}+O\left(\epsilon^{2}\right)$.

The boundary conditions at the particle surface, i.e., at $Y$ $=0$, are $u_{\theta}=U_{r}=0$. Let the gas-liquid interface be given by

$$
Y=H_{0}(\theta)+\epsilon H_{1}(\theta)+O\left(\epsilon^{2}\right) .
$$

Neglecting the gas density and the effect of gas flow on the liquid film, we have $p_{g}=0$ at the gas-liquid interface. For the zero interfacial tension case then, since the normal viscous stress is $O\left(\epsilon^{2}\right)$, the boundary condition for the liquid pressure is

$$
p=O\left(\epsilon^{2}\right) \quad \text { at } Y=H_{0}+\epsilon H_{1} .
$$

The normal and tangential vector perpendicular to the azimuthal direction at the gas-liquid interface are given by

$$
\mathbf{n}=\mathbf{e}_{r}-\epsilon H_{0}^{\prime} \mathbf{e}_{\theta}+O\left(\epsilon^{2}\right), \quad \mathbf{t}=\mathbf{e}_{\theta}+\epsilon H_{0}^{\prime} \mathbf{e}_{r}+O\left(\epsilon^{2}\right) .
$$

At low gas flow rates the tangential stress at the interface is negligible. Thus we have

$$
f_{t}^{*} \equiv \mathbf{e}_{\theta} \cdot \tau^{*} \cdot \mathbf{e}_{r}=\tau_{r \theta}+\epsilon\left(\tau_{r r}^{*}-\tau_{\theta \theta}^{*}\right)+O\left(\epsilon^{2}\right)=0,
$$

where $\tau^{*}$ is the dimensional stress tensor. Scaling stresses with $\mu_{l} U_{c} / \delta_{0}$, and noting that the stress components $\tau_{r r}^{*}$ and $\tau_{\theta \theta}^{*}$ are $O\left(\epsilon^{2}\right)$, we obtain $\tau_{r \theta}=0$ correct to $O\left(\epsilon^{2}\right)$ at the gas-liquid interface. This is equivalent to the boundary condition

$$
\frac{\partial u_{\theta}}{\partial Y}-\epsilon u_{\theta}=0 \quad \text { at } Y=H_{0}+\epsilon H_{1} .
$$

Expanding near $Y=H_{0}$, the above boundary condition reduces to

$$
\frac{\partial u_{\theta}}{\partial Y}+\epsilon\left(H_{1} \frac{\partial^{2} u_{\theta}}{\partial Y^{2}}-u_{\theta}\right)+O\left(\epsilon^{2}\right)=0 \quad \text { at } Y=H_{0} .
$$

The boundary condition of vanishing normal component of the velocity at the interface reduces to

$$
U_{r}-H_{0}^{\prime} u_{\theta}+O(\epsilon)=0 \quad \text { at } Y=H_{0} .
$$

Finally, scaling the liquid volumetric flow rate with $2 \pi a \rho_{l} g \delta_{0}^{3} /\left(3 \mu_{l}\right)$ we have the condition,

$$
\begin{aligned}
Q(\theta) & =3 \sin \theta \int_{0}^{H_{0}+\epsilon H_{1}} u_{\theta} d Y \\
& =3 \sin \theta\left[\int_{0}^{H_{0}} u_{\theta} d Y+\epsilon H_{1}+u_{\theta}\left(H_{0}\right)+O\left(\epsilon^{2}\right)\right],
\end{aligned}
$$

where $Q(\theta)$ equals unity for $0<\theta<\theta_{0}$ and $\left(\sin \theta / \sin \theta_{0}\right)^{2}$ for $0<\theta<\theta_{0}$ and $\pi-\theta_{0}<\theta<\pi$. 
The solution of the above set of equations is given by

$$
\begin{aligned}
u_{\theta}= & \sin \theta\left[Y H_{0}-Y^{2} / 2\right]+\epsilon\left[\operatorname { s i n } \theta \left\{\frac{Y^{3}}{2}-\frac{3 H_{0} Y^{2}}{2}\right.\right. \\
& \left.\left.+\left(2 H_{0}^{2}+H_{1}\right) Y\right\}+\cos \theta H_{0}^{\prime}\left\{\frac{Y^{2}}{2}-H_{0} Y\right\}\right]+O\left(\epsilon^{2}\right),
\end{aligned}
$$

$$
\begin{aligned}
& p=\cos \theta\left[H_{0}-Y\right]+O(\epsilon), \\
& U_{r}=2 \cos \theta\left(Y^{2} / 2-Y H_{0}\right)-\sin \theta Y H_{0}^{\prime}, \\
& H_{1}=-\frac{5 H_{0}^{2}}{8}+\frac{\cos \theta}{3 \sin \theta} H_{0} H_{0}^{\prime}, \\
& H_{0}=(\sin \theta)^{-2 / 3} \text { for } \theta_{0}<\theta<\pi-\theta_{0} .
\end{aligned}
$$

Near the north and south poles, $H_{0}=\left(\sin \theta_{0}\right)^{-2 / 3}$.

Now we derive the boundary conditions for the gas velocity. The continuity of the velocity at the gas-liquid interface gives

$$
\begin{aligned}
u_{\theta, g}\left(r=1+\epsilon H_{0}+\epsilon^{2} H_{1}\right)= & u_{\theta, l}\left(Y=H_{0}+\epsilon H_{1}\right) \\
= & \sin \theta H_{0}^{2} / 2+\epsilon\left\{\sin \theta\left(H_{0}^{3}+H_{1} H_{0}\right)\right. \\
& \left.-\cos \theta H_{0}^{2} H_{0}^{\prime} / 2\right\}+O\left(\epsilon^{2}\right),
\end{aligned}
$$

where $u_{\theta, g}$ and $u_{\theta, l}$ are, respectively, the gas and liquid angular velocities. Now expanding the gas velocity in powers of $\epsilon$, i.e., writing $u_{\theta, g}=u_{\theta, g}^{(0)}+\epsilon u_{\theta g}^{(1)}+\cdots$, we obtain

$$
\begin{aligned}
u_{\theta, g}^{(0)}(r=1)= & (1 / 2) \sin \theta H_{0}^{2}, \\
u_{\theta, g}^{(1)}(r=1)= & -H_{0}\left(\frac{\partial u_{\theta, g}^{(0)}}{\partial r}\right)_{r=1}+(3 / 8) \sin \theta H_{0}^{3} \\
& -(1 / 6) \cos \theta H_{0}^{2} H_{0}^{\prime} .
\end{aligned}
$$

The boundary condition for the other components of $\mathbf{u}_{g}^{(0)}$ and $\mathbf{u}_{g}^{(1)}$ may be similarly derived. The result, after expressing the quantities in the dimensional variables, is given in the main text.

\section{APPENDIX B: FILM THICKNESS IN THE CAPILLARY MODEL}

In the main text we presented an analysis for the film thickness and pressure drop that was valid for thin liquid films. The analysis for the case of arbitrary film thickness is straightforward. The resulting expressions are

$$
\begin{aligned}
(4 / 3) \epsilon_{0 c}^{3}= & \left(1-\left|\nabla p^{*}\right|\right)\left(\epsilon_{c}^{2}-\epsilon_{c}^{3}+\epsilon_{c}^{4} / 4\right) \\
& -\left(1-\epsilon_{c}\right)^{2}\left[\left(1-\epsilon_{c}\right)^{2} \ln \left(1-\epsilon_{c}\right)+\epsilon_{c}-\epsilon_{c}^{2} / 2\right],
\end{aligned}
$$

$$
\begin{aligned}
\left|\nabla p^{*}\right|= & \frac{8 \epsilon_{0 c} U_{c}^{*}}{\left(1-\epsilon_{c}\right)^{4}}\left[1+\frac{2 \mu_{g}}{\mu_{l}\left|\nabla p^{*}\right|}\left\{\frac{\epsilon_{c}\left(\epsilon_{c}-2\right)}{\left(1-\epsilon_{c}\right)^{2}}\right.\right. \\
& \left.\left.\times\left(1-\left|\nabla p^{*}\right|\right)-2 \ln \left(1-\epsilon_{c}\right)\right\}\right]^{-1} .
\end{aligned}
$$

The relation between the superficial liquid velocity and $\epsilon_{0 c}$ given by (49) is valid only for thin liquid films. For thicker films, the superficial liquid velocity can be computed from $\delta_{0 c}$ using

$$
\begin{aligned}
U_{l}= & \left(1-\phi_{s}\right) a_{c}^{2} \frac{\rho_{l} g}{2 \mu_{l}}\left[\epsilon_{0 c}^{2}-\epsilon_{0 c}^{3}+\epsilon_{0 c}^{4} / 4\right. \\
& \left.-\left(1-\epsilon_{0 c}\right)^{2}\left\{\left(1-\epsilon_{0 c}\right)^{2} \ln \left(1-\epsilon_{0 c}\right)+\epsilon_{0 c}-\epsilon_{0 c}^{2} / 2\right\}\right] .
\end{aligned}
$$

In the above, $\left|\nabla p^{*}\right|=|\nabla p| /\left(\rho_{l} g\right)+\rho_{g} / \rho_{l}$.

The pressure drop for a given liquid holdup can be determined using (B1). The corresponding gas velocity $U_{c}^{*}$ is subsequently determined from (B2) by substituting for $\nabla p^{*}$ and $\epsilon_{c}$.

${ }^{1}$ P. C. Carman, "Fluid flow through granular beds," Trans. Inst. Chem. Eng. 15, 150 (1937).

${ }^{2}$ S. Ergun, "Fluid flow through packed columns," Chem. Eng. Prog. 48, 93 (1952).

${ }^{3}$ J. P. Sorenson and W. E. Stewart, "Computation of forced convection in slow flow through ducts and packed beds. II. Velocity profile in simple cubic array of spheres," Chem. Eng. Prog. 29, 819 (1974).

${ }^{4}$ A. A. Zick and G. M. Homsy, "Stokes flow through periodic arrays of spheres," J. Fluid Mech. 115, 13 (1982).

${ }^{5}$ A. S. Sangani and A. Acrivos, "Slow flow through a periodic array of spheres,' Int. J. Multiphase Flow 8, 343 (1982).

${ }^{6}$ A. J. C. Ladd, "Hydrodynamic transport coefficients of random dispersions of hard spheres," J. Chem. Phys. 93, 3484 (1990).

${ }^{7}$ G. Mo and A. S. Sangani, "A method for computing Stokes flow interactions among spherical objects and its application to suspensions of drops and porous particles," Phys. Fluids 6, 1637 (1994).

${ }^{8} \mathrm{C}$. K. Ghaddar, "On the permeability of unidirectional fibrous media: A parallel computational approach," Phys. Fluids 7, 2563 (1995).

${ }^{9}$ D. L. Koch and A. J. C. Ladd, "Moderate Reynolds number flows through periodic and random arrays of aligned cylinders" J. Fluid Mech. 349, 31 (1997).

${ }^{10}$ D. C. Dankworth and S. Sundaresan, "A macroscopic model for countercurrent gas-liquid flow in packed beds," AIChE J. 35, 1282 (1989).

${ }^{11}$ K. A. Grosser, R. G. Carbonell, and S. Sundaresan, "The transition to pulsing flow in trickle beds," AIChE J. 34, 1850 (1988).

${ }^{12}$ D. C. Dankworth, I. G. Kevrekidis, and S. Sundaresan, "Dynamics of pulsing flow in trickle beds," AIChE J. 36, 605 (1990).

${ }^{13} \mathrm{~K}$. M. Ng, "A model for flow regime transitions in concurrent down-flow trickle-bed reactors," AIChE J. 32, 115 (1986)

${ }^{14}$ J. M. de Santos, T. R. Melli, and L. E. Scriven, "Mechanics of gas-liquid flow in packed-bed contactors," Annu. Rev. Fluid Mech. 23, 233 (1991).

${ }^{15}$ H. Lamb, Hydrodynamics (Dover, New York, 1945).

${ }^{16}$ D. L. Koch and A. S. Sangani, "Particle pressure and marginal stability limits for a homogeneous gas fluidized bed: Kinetic theory and numerical simulations," J. Fluid Mech. (to be submitted).

${ }^{17}$ A. E. Saez and R. G. Carbonell, "Hydrodynamic parameters for gas-liquid concurrent flow in packed beds," AIChE J. 31, 52 (1985).

${ }^{18}$ T. K. Sherwood and H. Shipley, "Flooding velocities in packed columns," Ind. Eng. Chem. 30, 765 (1938).

${ }^{19}$ B. E. T. Hutton, L. S. Leung, P. C. Brooks, and D. J. Nicklin, "On flooding in packed columns," Chem. Eng. Sci. 29, 493 (1974).

${ }^{20} \mathrm{~V}$. Specchia and G. Baldi, "Pressure drop and liquid holdup for two phase concurrent flow in packed beds," Chem. Eng. Sci. 32, 515 (1977). 